\title{
OKA'S PRINCIPLE FOR HOLOMORPHIC FIBER BUNDLES WITH SPRAYS
}

\author{
Franc Forstnerič and Jasna Prezelj
}

\section{\&1. The Oka-Grauert principle.}

The subject of this paper is the homotopy principle, also called the h-principle or the Oka-Grauert principle, concerning sections of certain holomorphic fiber bundles on Stein manifolds. We give a proof of a theorem of Gromov (1989) from sec. 2.9 in [Gro]; see theorems 1.3 and 1.4 below. This result, which extends the work of H. Grauert from 1957 ([Gr3], [Gr4], [Car]), has been used in the proofs of the embedding theorem for Stein manifolds into Euclidean spaces of minimal dimension [EGr], [Sch].

1.1 Definition. Let $h: Z \rightarrow X$ be a holomorphic mapping of complex manifolds. A section of $h$ is any map $f: X \rightarrow Z$ such that $h \circ f$ is the identity on $X$. We say that sections of $h$ satisfy the h-principle (or the Oka-Grauert principle) if each continuous section $f_{0}: X \rightarrow Z$ can be deformed to a holomorphic section $f_{1}: X \rightarrow Z$ through a continuous one parameter family (a homotopy) of continuous sections $f_{t}: X \rightarrow Z$ (0 $\leq t \leq 1$ ), and any two holomorphic sections $f_{0}, f_{1}: X \rightarrow Z$ which are homotopic through continuous sections are also homotopic through holomorphic sections. If this holds for a trivial bundle $Z=X \times F \rightarrow X$, we say that maps $X \rightarrow F$ satisfy the h-principle.

1.2 Definition. (Gromov [Gro]) A (dominating) spray on a complex manifold $F$ is a holomorphic vector bundle $p: E \rightarrow F$, together with a holomorphic map $s: E \rightarrow F$, such that $s$ is the identity on the zero section $F \subset E$, and for each $x \in F$ the derivative $D s(x)$ maps $E_{x}$ (which is a linear subspace of $T_{x} E$ ) surjectively onto $T_{x} F$.

The following result can be found in sec. 2.9 of [Gro].

1.3 Theorem. If $F$ is a complex manifold which admits a spray, then the sections of any locally trivial holomorphic fiber bundle with fiber $F$ over any Stein manifold satisfy the h-principle. In particular, mappings from Stein manifolds into $F$ satisfy the h-principle.

Stronger results are given in theorem 1.4 and corollary 1.5 below. In the sequel [FP] to this paper we give a proof of Gromov's Main Theorem ([Gro], sect. 4.5) to the effect that the h-principle holds for sections of holomorphic submersions $h: Z \rightarrow X$, where $X$ is Stein and each point $x \in X$ has a neighborhood $U \subset X$ such that $Z \mid U=h^{-1}(U)$ admits a fiber-spray (see def. 3.1 below).

For non-specialists we recall that a complex manifold is called Stein (after Karl Stein, 1951 [Ste]) if it has 'plenty' of global holomorphic functions. For the precise definition 
and properties we refer the reader to the monographs [GRo], [Hö2] and [GRe]. The most commonly used characterizations are the following. A complex manifold $X$ is Stein if and only if any of the following two conditions holds:

- $X$ can be embedded as a closed complex submanifold of some complex Euclidean space (the embedding theorem of Remmert, Bishop, and Narasimhan [GRo, p. 224]);

- $X$ admits a smooth strongly plurisubharmonic exhaustion function (Grauert [Gr1]).

By a locally trivial holomorphic fiber bundle with fiber $F$ over a complex manifold $X$ we mean a bundle obtained by patching the trivial bundles $U_{\alpha} \times F$ over an open covering $\left\{U_{\alpha}\right\}$ of $X$ by transition functions of the form

$$
\phi_{\alpha, \beta}(x, \xi)=\left(x, \psi_{\alpha, \beta}(x, \xi)\right) \quad\left(x \in U_{\alpha, \beta}=U_{\alpha} \cap U_{\beta}, \xi \in F\right),
$$

where $\psi_{\alpha, \beta}: U_{\alpha, \beta} \times F \rightarrow F$ is holomorphic and $\psi_{\alpha, \beta}(x, \cdot) \in \operatorname{Aut} F$ is an automorpism of $F$ for each fixed $x$. Of course these transition function must satisfy the usual compatibility conditions, see for instance [Car].

Examples of bundles satisfying theorem 1.3 include those whose fiber is a complex Lie group $L$ or an $L$-homogeneous space; see examples (A) and (B) below for the existence of spray. The simplest bundles of this type are the principal holomorphic $L$-bundles in which the transition maps are given left multiplications by holomorphic $L$-valued functions $U_{\alpha, \beta} \rightarrow L$, and $L$ acts on each fiber $E_{x}$ by right multiplication. Another example are bundles in which the transition maps $\psi_{\alpha, \beta}(x, \cdot)$ are Lie group automorphisms of $L$. In these cases the h-principle had been proved by Grauert [Gr3], [Car].

In general the automorphisms $\psi_{\alpha, \beta}(x, \cdot)$ need not preserve any additional structure the fiber might have and hence we get much more general fiber bundles. In this connection we recall an important example of Demailly [Dem]:

Example 1. There exists a locally trivial holomorphic fiber bundle $h: Z \rightarrow X$, with base $X$ either $\mathbf{C}$ or the disc and with fiber $\mathbf{C}^{2}$, such that $Z$ has no holomorphic functions other than those of the form $g \circ h$, where $g$ is holomorphic on the base $X$. So $Z$ is not Stein. In particular, $Z$ admits no holomorphic vector bundle structure which gives a negative answer to the question of Gromov [Gro, 2.5.B]. The transition functions used in the construction of $Z$ are (nonlinear) holomorphic automorphisms of the fiber $\mathbf{C}^{2}$. Nevertheless the sections of $Z \rightarrow X$ satisfy the h-principle according to theorem 1.3.

One of the most important sources of spaces with sprays is the following [Gro]:

(*) If $F$ is a complex manifold which admits finitely many $\mathbf{C}$-complete holomorphic vector fields $V_{j}, 1 \leq j \leq J$, such that the vectors $V_{j}(x)$ span the tangent space $T_{x} F$ at each point $x \in F$, then $F$ admits a spray.

Recall that a holomorphic vector field is $\mathbf{C}$-complete if for any $x \in F$ the flow $t \rightarrow \phi^{t}(x)$ of $V$, with $\phi^{0}(x)=x$, is defined for all complex values $t \in \mathbf{C}$ of the time parameter. (For a discussion of $\mathbf{R}$-completeness versus $\mathbf{C}$-completeness see [Fo1] and [AFR].) Indeed, if we denote by $\phi_{j}^{t}$ the flow of $V_{j}$ on $F$ and let $s: F \times \mathbf{C}^{J} \rightarrow F$ be given by

$$
s\left(x ; t_{1}, \ldots, t_{J}\right)=\phi_{1}^{t_{1}} \circ \phi_{2}^{t_{2}} \circ \cdots \circ \phi_{J}^{t_{J}}(x),
$$


we have $s(x ; 0, \ldots, 0)=x$ and $\frac{\partial}{\partial t_{j}} s(x ; 0, \ldots, 0)=V_{j}(x)(x \in F, 1 \leq j \leq J)$. If these vectors span $T_{x} F$ for each $x \in F, s$ is a spray on $F$.

We list some examples of complex manifolds with sprays:

(A) Any complex Lie group $L$. (Take any finite set of left or right invariant vector fields on $L$ which span $T_{e} L=$ the Lie algebra of $L$.)

(B) Let $L$ be a complex Lie group with Lie algebra $\ell$. If $L$ acts holomorphically and transitively on a complex manifold $F$, the map $s: F \times \ell \rightarrow F, s(x, l)=\exp (l) x$ $(x \in F, l \in \ell)$, is a spray on $F . F$ is then biholomorphic to the $L$-homogeneous space $L / H=\{l H: l \in L\}$ where $H$ is a complex Lie subgroup of $L$ (the isotropy group of a point in $Y$ ).

(C) $F=\mathbf{C}^{n} \backslash \Sigma$, where $\Sigma$ is an algebraic subvariety of complex codimension at least two (see below).

We emphasize that, at the time of this writing, the known proofs of the embedding theorem for Stein manifolds (and Stein spaces) to Euclidean spaces of minimal dimension depend on the validity of the h-principle in the cases (B) and $(C)$ (see Eliashberg and Gromov [EGr] and Schürmann [Sch]).

In case (C) we think of $\Sigma$ as a set which should be avoided by the image of a map $X \rightarrow \mathbf{C}^{n}$. Theorem 1.3 asserts that we can avoid $\Sigma$ by a holomorphic map $f: X \rightarrow \mathbf{C}^{n}$ if we can do so by a continuous map. In this case we can obtain complete vector fields on $F$ of the form $V(z)=f(\pi(z)) v$ (shear fields), where $v \in \mathbf{C}^{n} \backslash\{0\}, \pi: \mathbf{C}^{n} \rightarrow \mathbf{C}^{n-1}$ is a linear projection with $\pi(v)=0$, and $f: \mathbf{C}^{n-1} \rightarrow \mathbf{C}$ is an entire function which vanishes on the projected set $\Sigma^{\prime}=\pi(\Sigma) \subset \mathbf{C}^{n-1}$. The flow of $V$, given by $\phi^{t}(z)=z+t f(\pi(z)) v$, fixes $\Sigma$ and hence induces a complete flow on $\mathbf{C}^{n} \backslash \Sigma$. The projection $\pi$ must be chosen such that it is proper when restricted to $\Sigma$ to insure that $\pi(\Sigma)=\Sigma^{\prime}$ is a proper closed subvariety of $\mathbf{C}^{n-1}$ of codimension at least one. This is true for most projections, see e.g. [Chi].

The argument above works as long as there exist sufficiently many linear projection $\pi: \mathbf{C}^{n} \rightarrow \mathbf{C}^{n-1}$ which are proper when restricted to $\Sigma$. The following example shows that one cannot completely dispose of the last condition.

Example 2. For any integer $N>0$ there exist discrete sets $\Sigma \subset \mathbf{C}^{N}$ for which there exist no non-degenerate holomorphic maps $\mathbf{C}^{N} \rightarrow \mathbf{C}^{N} \backslash \Sigma[\mathrm{RRu}$. Furthermore, for any $1 \leq n<N$ there exist proper holomorphic embeddings $\sigma: \mathbf{C}^{n} \rightarrow \mathbf{C}^{N}$ such that $\mathbf{C}^{N} \backslash \sigma\left(\mathbf{C}^{n}\right)$ admits no non-degenerate holomorphic images of $\mathbf{C}^{N-n}$ [BFo], [Fo2], and hence $\mathbf{C}^{N} \backslash \sigma\left(\mathbf{C}^{n}\right)$ admits no spray. In $[\mathrm{FP}]$ we show that the h-principle fails in these cases.

In these constructions, as in the work of Grauert [Gr3] and Gromov [Gro], one must consider not a single section at a time but families of sections, depending continuously on a parameter $y$ in a compact Hausdorff space $Y$. The basic objects will be continuous maps $f: X \times Y \rightarrow Z$ such that $f(\cdot, y): X \rightarrow Z$ is a section of $h: Z \rightarrow X$ for each fixed $y \in Y$. A homotopy of such maps is a continuous map $H: X \times Y \times[0,1] \rightarrow Z$ such that $H_{t}(\cdot, y)=H(\cdot, y, t): X \rightarrow Z$ is a section of $h: Z \rightarrow X$ for all $y \in Y$ and $t \in[0,1]$. 
Recall that a compact subset $K \subset X$ is holomorphically convex in $X$ if for each $x \in X \backslash K$ there is a holomorphic function $f$ on $X$ such that $|f(x)|>\sup _{K}|f|$. If $X$ is Stein then by the Oka-Weil theorem each function holomorphic in a neighborhood of a holomorphically convex set $K \subset X$ can be approximated on $K$ by functions holomorphic on $X[\mathrm{Hö} 2]$.

Whenever we use a metric on a manifold, we always mean a metric compatible with the underlying manifold topology. We shall not mention this again.

The next result is the parametric h-principle with approximation on holomorphically convex sets. A discussion in this direction can be found in sect. 3 in [Gro].

1.4 Theorem. Let $X$ be a Stein manifold and $h: Z \rightarrow X$ a locally trivial holomorphic fiber bundle whose fiber admits a spray. Let $Y$ be a compact Hausdorff space (the parameter space), $Y_{0} \subset Y$ a compact subset and $Y^{\prime} \subset Y$ an open set containing $Y_{0}$. Assume that $f: X \times Y \rightarrow Z$ is a continuous map such that $f(\cdot, y): X \rightarrow Z$ is a section of $h: Z \rightarrow X$ for each $y \in Y$, and $f(\cdot, y)$ is holomorphic on $X$ for each $y \in Y^{\prime}$. Then there is a homotopy $H: X \times Y \times[0,1] \rightarrow Z$ such that $H_{0}=f, H_{1}(\cdot, y): X \rightarrow Z$ is holomorphic on $X$ for each $y \in Y$, and the homotopy is fixed on $Y_{0}$ (i.e., $H_{t}(x, y)$ is independent of $t$ for $y \in Y_{0}$ ). Moreover, if $K$ is a compact holomorphically convex subset in $X$ and we assume that there is a neighborhood $V \subset X$ of $K$ such that each section $f(\cdot, y)(y \in Y)$ is holomorphic in $V$, then for any metric $d$ on $Z$ and for any $\epsilon>0$ there is a homotopy $H$ as above which also satisfies

$$
d\left(H_{t}(x, y), f(x, y)\right)<\epsilon \quad(x \in K, y \in Y, 0 \leq t \leq 1)
$$

Example 3. Theorem 1.4 fails for maps of any Stein manifold $X$ into any Kobayashihyperbolic complex manifold $F$. To see this, take $K$ to be a small piece of an embedded analytic disc in $X$ and let $f_{0}: K \rightarrow F$ be a holomorphic map which is close to being extremal with respect to the Kobayashi metric on $F$. Such $f_{0}$ cannot be approximated on $K$ by holomorphic maps $f: X \rightarrow F$ since this would create much larger analytic discs in $F$. Of course a hyperbolic manifold admits no spray since it even admits no non-degenerate holomorphic images of $\mathbf{C}$.

1.5 Corollary. If $h: Z \rightarrow X$ is as in theorem 1.3, the inclusion map between the spaces of holomorphic and continuous sections, $\operatorname{Holo}(X, Z) \hookrightarrow \operatorname{Cont}(X, Z)$, is a weak homotopy equivalence, i.e., it induces an isomorphism of the homotopy groups of the two spaces (endowed with the usual compact-open topology.)

Remarks. 1. The basic h-principle (def. 1.1) is equivalent to saying that each path connected component of the space of continuous sections $\operatorname{Cont}(X, Z)$ contains precisely one path connected component of the space of holomorphic sections $\operatorname{Holo}(X, Z)$.

2. If the set $Y_{0}$ in theorem 1.4 is a deformation retraction of some open neighborhood $Y^{\prime} \subset Y$, it suffices to assume only that the sections $f(\cdot, y)$ for $y \in Y_{0}$ are holomorphic on $X$ since we can use the deformation retraction to suitably reparametrize the family. In all our applications $Y$ will be a polyhedron and $Y_{0}$ a subpolyhedron. 
Proof of corollary 1.5. If we take $Y$ to be the $n$-sphere $S^{n}$ and $Y^{\prime}=\emptyset$, theorem 1.4 implies that each (continuous) map $S^{n} \rightarrow \operatorname{Cont}(X, Z)$ can be homotopically deformed to a map $S^{n} \rightarrow \operatorname{Holo}(X, Z)$. Similarly, if we take $Y$ to be the closed real $(n+1)$-ball $B^{n+1}$ and $Y_{0}=\partial B^{n+1}=S^{n}$, we conclude that each map $S^{n} \rightarrow \operatorname{Holo}(X, Z)$ which extends to a map $B^{n+1} \rightarrow \operatorname{Cont}(X, Z)$ also extends to a map $B^{n+1} \rightarrow \operatorname{Holo}(X, Z)$. This is precisely the content of corollary 1.5 .

We wish to put these results in historic perspective as we see it. The term Oka's principle can vaguely be stated by saying that, on a Stein manifold or a reduced Stein space, any analytic problem with no topological obstruction has an analytic solution. To be more precise we quote from p. 145 in [GRe]: Analytic problems which can be cohomologically formulated have only topological obstructions. Early examples include Oka's classification of holomorphic line bundles and the solvability of the second Cousin problem [Hö2], p. 144. There are also examples of this principle on non-Stein manifolds, such as the Riemann-Roch theorem.

A major extension of Oka's principle was obtained by H. Grauert in a series of deep and influential papers [Gr2]-[Gr4] in 1957-58; see also Cartan [Car] for a nice exposition of Grauert's work. The main result of Grauert [Gr3] was the proof of theorems 1.3 and 1.4 above for holomorphic fiber bundles $E \rightarrow X$ over Stein spaces $X$ (possibly with singularities), where the fiber $L$ is a complex Lie group and the transition maps $\psi_{\alpha, \beta}(x, \cdot)$ are Lie group automorphisms of $L$. Each fiber $E_{x}$ therefore carries a natural Lie group structure isomorphic to $L$, and there is a well defined identity section of $E$. The (flows of) left invariant holomorphic vector fields on $L$ induce a spray on $L$, and hence theorem 1.3 includes Grauert's theorem. In fact there exists even a global spray on $E$, induced by flows of left invariant fields on $E$ tangent to the fibers $E_{x}$.

Cartan [Car] observed that Grauert's proof carries over immediately to fiber bundles $\tilde{E} \rightarrow X$ over a Stein base, where $E \rightarrow X$ is as above and where each fiber $\tilde{E}_{x}$ is a $E_{x^{-}}$ homogeneous space, i.e., the elements of $E_{x} \simeq L$ act on $\tilde{E}_{x}$ by right multiplication. Cartan calls such $\tilde{E}$ an espace analytique E-principal. (Cartan's observation was acknowledged by Grauert in the sentence preceeding his Satz 2 in [Gr3] on p.267.) When $E \simeq X \times L$ is a trivial bundle, $\tilde{E}$ is a classical principal $L$-bundle. The validity of the h-principle for such bundles $\tilde{E}$ implies that the holomorphic classification of the principal holomorphic bundles over a Stein space agrees with the topological classification [Car, Gr4]. This holds in particular for holomorphic vector bundles. Further results were obtained by Ramspott [Ram], Forster and Ramspott [FR1, FR2], Forster [For], Heinzner and Kutzschebauch $[\mathrm{HKu}]$, and others.

Grauert's constructions were similar in spirit to the usual proof of Cartan's theorems $\mathrm{A}$ and $\mathrm{B}$ for coherent analytic sheaves. The local patching of holomorphic sections on small sets was obtained (like the 'patching of syzygies') by an analogue of the Cartan's splitting lemma for holomorphic matrices. The globalization followed the usual scheme of passing from one compact Stein block in $X$ to the next block. (A Stein block is a compact set in $X$ that can be embedded as a closed complex subvariety of a cube in a Euclidean space $\mathbf{C}^{N}$ by a holomorphic map $X \rightarrow \mathbf{C}^{N}$.) To insure the convergence one must correct the section at each step by a suitable version of the Runge approximation theorem which 
had been established in [Gr2].

This scheme does not seem to carry over to more general fiber bundles because no simple proof of the Runge theorem is available. Things didn't progress very much until 1986 when Henkin and Leiterer ([HL2], [HL3]) introduced the Grauert's bump method to this problem and gave a conceptually new proof of Grauert's theorem. This method had been used earlier in the solution of the Levi problem [HL1]; we shall comment on it more extensively below. The crucial point in this approach is that the Runge theorem is only needed for sections on small compact subsets of $X$. Possibly the only shortfall is that, so far at least, the method only applies to nonsingular Stein bases.

In 1989 M. Gromov [Gro] made a crucial step by replacing the exponential map in fibers by the much more flexible concept of a spray. This made it possible to extend the results to the present form.

When reading sect. 2 in [Gro] we were unable to complete the proof as suggested there due to seemingly nontrivial analytic and geometric problems. Eventually we completed the proof in a different way, using the work of Henkin and Leiterer [HL3] and the tools from [Gro]. We give here a complete exposition of our version of the proof. We also take this opportunity to provide a detailed account, with proofs, of all major tools.

In the rest of this section we explain the outline of our proof and also point out the difficulties that we had with sect. 2 in [Gro]. For the sake of simplicity we only discuss the simplest case: to deform a continuous section by a homotopy to a holomorphic section.

The underlying geometric scheme is the Grauert's 'bump method' (sect. 2); our reference for this is [HL3]. The manifold $X$ is exhausted by an increasing family of smooth, compact, strongly pseudoconvex domains $A_{k} \subset \subset X$ such that $A_{k+1}=A_{k} \cup B_{k}$, where $B_{k}$ is a small strongly pseudoconvex domain attached to $A_{k}$ in a certain special way (a special pseudoconvex bump in the terminology of [HL3]; see def. 2.6 there or def. 2.2 in the present paper). We may assume that the bundle $Z$ is trivial over a neighborhood of $B_{k}$. To obtain such an exhaustion one begins with a smooth strongly plurisubharmonic exhaustion function $\rho: X \rightarrow \mathbf{R}$ with nice critical points. One can pass from one sublevel set $\{\rho \leq c\}$ to a higher sublevel set $\left\{\rho \leq c^{\prime}\right\}$, where $c<c^{\prime}$ are regular values of $\rho$, by attaching a small 'bump' $B_{k}$ at each step. These bumps are contractible strongly pseudoconvex domains such that $C_{k}=A_{k} \cap B_{k}$ is also contractible, except when passing a critical point of $\rho$ when $C_{k}$ becomes a torus. Moreover, $C_{k}$ is Runge in $B_{k}$.

Granted such an exhaustion of $X$, we inductively construct a sequence of continuous sections $f_{k}: X \rightarrow Z$ such that $f_{k}$ is holomorphic in a neighborhood of $A_{k}$, it approximates $f_{k-1}$ on $A_{k-1}$, and is homotopic to the original section $f_{0}$. The desired holomorphic section is then obtained as the locally uniform limit $f=\lim _{k \rightarrow \infty} f_{k}: X \rightarrow Z$.

Suppose $f_{k}$ as above has been constructed. To construct $f_{k+1}$ we proceed as in [Gro] or [HL3]. Since $Z$ is trivial over $B_{k}$, there is a holomorphic section $b_{0}: B_{k} \rightarrow Z$ homotopic to $f_{0}$. The first step is to construct a holomorphic homotopy $b_{t}: C_{k} \rightarrow Z, 0 \leq t \leq 1$, connecting $b_{0}$ and $b_{1}=f_{k} \mid C_{k}$ over $C_{k}$. This is the difficult part and it is here that our proof differs from [Gro]. We shall comment on this later. From now on the process follows [Gro]. Since $b_{0}$ is holomorphic on $B_{k}$, and $C_{k}$ is Runge in $B_{k}$, the homotopy version of Runge theorem (sect. 4) allows us to approximate the homotopy $b_{t}$ uniformly on $C_{k}$ by 
a holomorphic homotopy $\tilde{b}_{t}$ defined on $B_{k}$. The section $b=\tilde{b}_{1}$ approximates $f_{k}$ as well as desired on $C_{k}$, so we can glue them (see sect. 5) into a single section $f_{k+1}$ that is holomorphic on $A_{k+1}=A_{k} \cup B_{k}$ and approximates $f_{k}$ on $A_{k}$. It remains to extend $f_{k+1}$ continuously to $X$ so that it is homotopic to $f_{0}$. In the process we must also insure the convergence of homotopies from $f_{0}$ to $f_{k}$ on $X$.

Back to the homotopy $b_{t}$ on $C_{k}$. In the non-critical case the set $C_{k}=A_{k} \cap B_{k}$ is holomorphically contractible. Since $Z$ is trivial over $C_{k}$, we immediately get the required homotopy by using the contractions of $C_{k}$ to a point. This non-critical case allows us to proceed in a finite number of steps from a sublevel set $\left\{\rho \leq c_{0}\right\}$ to a higher sublevel set $\left\{\rho \leq c_{1}\right\}$, provided that $\rho$ has no critical values on the interval $\left[c_{0}, c_{1}\right] \subset \mathbf{R}$. A similar method (with $C_{k}=\emptyset$ ) allows us to cross the critical points of $\rho$ which are local minima.

To pass a critical point $x_{0}$ of $\rho$ which is not a local minimum, Gromov suggested (sec. 2.7 in [Gro]) to attach to the set $A_{k}=\{\rho \leq c\}$, for some $c<c_{0}=\rho\left(x_{0}\right)$ and $c$ close to $c_{0}$, a real-analytic, totally real disc $B_{k}$ containing $x_{0}$. We must then find small strongly pseudoconvex neighborhoods $V \subset X$ of $A_{k} \cup B_{k}$ such that we can reach a suitable higher sublevel set $\left\{\rho \leq c^{\prime}\right\}$ for $c^{\prime}>c_{0}$ by attaching convex bumps to $V$ (i.e., as a noncritical pseudoconvex extension of $V$ ). If we understand correctly, the idea in [Gro] was to take a homotopy $\left\{b_{t}^{\prime}\right\}$ from $b_{0}^{\prime}=b_{0}$ to $b_{1}^{\prime}=f_{k}$ over the disc $B_{k}$ (such exist since $B_{k}$ is contractible), then approximate it by a real-analytic homotopy $b_{t}$ on $B_{k}$, and finally complexify $b_{t}$ to get a holomorphic homotopy whose final section $b=b_{1}$ approximates $f_{k}$ on (and hence near) the totally real collar $C_{k}=A_{k} \cap B_{k}$. It was then suggested to proceed with gluing as before.

We could not complete this for two reasons. The first problem is to find suitable neighborhoods $V$ of $A_{k} \cup B_{k}$ with the required properties. We do not know of any such result in the literature; for partial results see [Eli] and [Ros]. Another independent problem is that the rate of approximation of $f_{k}$ by $b$, required to glue them into a single section, depends on $V$ since we must solve a certain $\bar{\partial}$-equation with sup norm estimates in the process. Even though this can be done on any strongly pseudoconvex domain, the constant in such estimate depends (unlike for the $L^{2}$ estimate!) on the geometry of the set. If the sets degenerate, the constants will blow up in general. If on the other hand we work with the $L^{2}$ estimates, the loss in the Cauchy estimates again depends on the shape of $V$. However, we do not see how to control the rate of approximation of $f_{k}$ by $b$ in any fixed neighborhood of the collar $A_{k} \cap B_{k}$ when $b$ is obtained as above. See also remark 1 following the proof of lemma 2.4 below.

We instead proceed as follows. Suppose that $x_{0} \in X$ is a critical point of $\rho$. Set $c_{0}=\rho\left(x_{0}\right)$. It was proved in [HL3] that, if $c<c_{0}$ and $c$ is sufficiently close to $c_{0}$, we can attach to the set $A_{k}=\{x \in X: \rho(x) \leq c\}$ a pseudoconvex bump $B_{k}$ as follows. The set $C_{k}=A_{k} \cap B_{k}$ is a sublevel set $\{\tau \leq 1\}$ of some strongly plurisubharmonic function $\tau \geq 0$, defined in a neighborhood of $B_{k}$, such that (in some holomorphic coordinate system in a neighborhood of $B_{k}$ ) the set $S=\tau^{-1}(0)$ is a sphere contained in an affine totally real subspace, and $\tau$ has no critical points on $C_{k} \backslash S$. In particular, $C_{k}$ is obtained from a small tubular neighborhood of $S$ by attaching convex bumps. Furthermore, a suitable sublevel set $\left\{\rho \leq c^{\prime}\right\}$ for some $c^{\prime}>c_{0}$ is a non-critical strongly pseudoconvex extension of $A_{k+1}=A_{k} \cup B_{k}$. 
To construct the homotopy $b_{t}$ over $C_{k}$ we initially deform a given continuous homotopy $b_{t}^{\prime}$ from $b_{0}^{\prime}=b_{0}$ to $b_{1}^{\prime}=f_{k}$ (which exists on $B_{k}$ ) in a small tube around the sphere $S$ to make it holomorphic there. This is possible since $S$ is totally real. Subsequently we extend it to $C_{k}$ in a finite number of steps. In each step we extend the homotopy (by approximation) across a convex bump, using parametric versions of the h-principle and of the gluing lemma. Since $C_{k}$ is a non-critical extension of a tube around $S$, this allows us to complete the proof of theorems 1.3 and 1.4. The crucial new steps in our proof are theorem 4.5 and corollary 5.6.

The paper is organized as follows. In section 2 we recall the bump method, following [HL3], and we reduce the proof of theorem 1.4 to theorem 2.6 concerning the extension of holomorphic sections across pseudoconvex bumps. Theorem 2.6 is proved in sect. 6 after we develop the necessary tools in sect. 3-5. In sect. 3 we recall from [Gro] the relevant properties of sprays and iterated spray bundles, and we prove that any homotopy of holomorphic sections of $Z \rightarrow X$ can be lifted to a homotopy of sections of an iterated spray bundle over $Z$. In sect. 4 we prove Runge-type approximation theorems for sections of holomorphic submersions which admit a spray. In sect. 5 we prove results on gluing holomorphic sections over Cartan pairs. In sect. 6 we complete the proof of theorem 2.6.

We wish to thank G. Henkin and J. Leiterer for their interest in our work and for several useful discussions. We also thank J. Globevnik who got us interested in this topic, and the participants in the Seminar for complex analysis at the University of Ljubljana who suffered through our lectures. The first author acknowledges partial support by the National Science Foundation, by the Vilas Foundation at the University of Wisconsin, and by the Ministry of Science and Technology of the Republic of Slovenia. The second author was supported by the Ministry of Education of the Republic of Slovenia.

\section{\&2. Pseudoconvex bumps and Cartan pairs.}

The main reference for this section is [HL3]. Let $X$ be a complex manifold. We say that a compact set $C \subset X$ is Runge in another compact set $B \supset C$ if $C$ has a basis of neighborhoods $C_{j}$ which are Runge in some open neighborhood $\tilde{B}$ of $B$, i.e., each function holomorphic in $C_{j}$ can be approximated uniformly on compacts in $C_{j}$ by functions holomorphic in $\tilde{B}$. This is the case for instance if $C$ is holomorphically convex in some Stein neighborhood of $B$.

2.1 Definition. Let $X$ be a complex manifold.

(i) A compact strongly pseudoconvex domain in $X$ is a compact set of the form $A=$ $\{\rho \leq 0\} \subset X$, where $\rho: X \rightarrow \mathbf{R}$ is a $\mathcal{C}^{2}$ function which is strongly plurisubharmonic in a neighborhood of $\{\rho=0\}$ and has no critical points on $\{\rho=0\}$.

(ii) Let $A \subset A^{\prime}$ be a pair of compact strongly pseudoconvex domains in $X$. We say that $A^{\prime}$ is a strongly pseudoconvex extension of $A$ in $X$ if there is a $\mathcal{C}^{2}$ function $\rho: X \rightarrow \mathbf{R}$ which is strongly plurisubharmonic on $\overline{A^{\prime} \backslash A}$ such that, for some real numbers $\beta>\alpha$ which are regular values of $\rho$,

$$
A=\{x \in X: \rho(x) \leq \alpha\}, \quad A^{\prime}=\{x \in X: \rho(x) \leq \beta\} .
$$


If $\rho$ can be chosen such that it has no critical points on $\overline{A^{\prime} \backslash A}, A^{\prime}$ is called a noncritical strongly pseudoconvex extension of $A$.

2.2 Definition. Let $X$ be a complex manifold. A pair $(A, B)$ of compact subsets of $X$ is a pseudoconvex bump (or $B$ is a pseudoconvex bump on $A$ ) if the following hold:

(i) The sets $A, B, A \cup B$ and $C=A \cap B$ are compact strongly pseudoconvex domains ( $C$ may be empty);

(ii) $\overline{A \backslash B} \cap \overline{B \backslash A}=\emptyset$;

(iii) there are holomorphic coordinates in a neighborhood of $B$ in which $B$ is star-shaped;

(iv) $C$ is Runge in $B$;

(v) if $C \neq \emptyset$ there is a $\mathcal{C}^{2}$ strongly plurisubharmonic function $\tau \geq 0$ defined in a neighborhood $U \subset X$ of $B$ such that $C=\{z \in U: \tau(z) \leq 1\}$, the set $S=\tau^{-1}(0)$ is a compact totally real submanifold contained in an affine totally real subspace (with respect to some holomorphic coordinates in a neighborhood of $B$ ), and $\tau$ has no critical points in $C \backslash S=\{z \in U: 0<\tau(z) \leq 1\}$.

We say that $B$ is a convex bump on $A$ (or the pair $(A, B)$ is a convex bump) if, in addition to the above, there are holomorphic coordinates in a neighborhood of $B$ in which both $B$ and $C$ are strongly convex domains. (The set $C$ may be empty.)

For certain purposes we can relax the above conditions and consider Cartan pairs. Gromov's definition of a Cartan pair is rather imprecise (sec. 1.5.A in [Gro]); we shall adopt the following definition.

2.3 Definition. A Cartan pair in $X$ is a pair of compact sets $A, B \subset X$ such that

(i) $A, B$, and $A \cup B$ have bases of Stein neighborhoods, and

(ii) $\overline{A \backslash B} \cap \overline{B \backslash A}=\emptyset$.

Any pseudoconvex bump is clearly a Cartan pair. We denote by $H^{\infty}(\Omega)$ the algebra of bounded holomorphic functions on $\Omega$. The following lemma will be used in sec. 5 for gluing holomorphic sections over Cartan pairs; this is similar to what Gromov takes as the definition of a Cartan pair.

2.4 Lemma. If $(A, B)$ is a Cartan pair in a Stein manifold such that $C=A \cap B \neq \emptyset$, there are bases of Stein open neighborhoods $A_{j} \supset A, B_{j} \supset B, C_{j}=A_{j} \cap B_{j} \supset A \cap B$, and bounded linear operators $\mathcal{A}_{j}: H^{\infty}\left(C_{j}\right) \rightarrow H^{\infty}\left(A_{j}\right), \mathcal{B}_{j}: H^{\infty}\left(C_{j}\right) \rightarrow H^{\infty}\left(B_{j}\right)$, satisfying

$$
c=\mathcal{A}_{j}(c)-\mathcal{B}_{j}(c), \quad c \in H^{\infty}\left(C_{j}\right), j=1,2,3, \ldots
$$

Proof. Let $U \supset A$ and $V \supset B$ be open neighborhoods of $A$ resp. $B$. Choose Stein open sets $\tilde{A}$ and $\tilde{B}$ in $X$ so that $A \subset \tilde{A} \subset U$ and $B \subset \tilde{B} \subset V$. Set $\tilde{C}=\tilde{A} \cap \tilde{B}$. By the separation

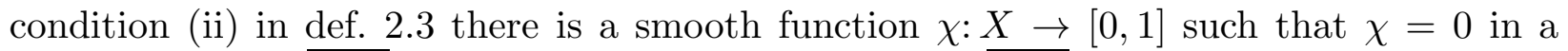
neighborhood of $\overline{A \backslash B}$ and $\chi=1$ in a neighborhood of $\overline{B \backslash A}$. Hence there are open sets 
$A_{0}, B_{0} \subset X$, with $A \subset A_{0} \subset \tilde{A}$ and $B \subset B_{0} \subset \tilde{B}$, such that $\chi=0$ on $A_{0} \backslash \tilde{B}$ and $\chi=1$ on $B_{0} \backslash \tilde{A}$. Choose a smooth strongly pseudoconvex set $\Omega$, with $A \cup B \subset \Omega \subset A_{0} \cup B_{0}$, and set

$$
A^{\prime}=\tilde{A} \cap \Omega, \quad B^{\prime}=\tilde{B} \cap \Omega, \quad C^{\prime}=A^{\prime} \cap B^{\prime}=\tilde{C} \cap \Omega .
$$

Then $A^{\prime}, B^{\prime}$ and $C^{\prime}$ are Stein domains containing $A, B, C$ respectively, with $A^{\prime} \subset \tilde{A} \subset U$, $B^{\prime} \subset \tilde{B} \subset V$, and $A^{\prime} \cup B^{\prime}=\Omega$. Moreover by the choice of $\Omega$ we have

$$
A^{\prime} \backslash C^{\prime}=A^{\prime} \backslash B^{\prime}=\Omega \backslash \tilde{B} \subset A_{0} \backslash \tilde{B} \subset\{\chi=0\}
$$

and similarly $B^{\prime} \backslash C^{\prime} \subset\{\chi=1\}$. If $c$ is any bounded holomorphic function in $C^{\prime}$ then the above implies that $\chi c$ extends to a bounded smooth function in $A^{\prime}$ which vanishes in $A^{\prime} \backslash C^{\prime},(1-\chi) c$ extends to a bounded smooth function in $B^{\prime}$ which vanishes in $B^{\prime} \backslash C^{\prime}$, and $\bar{\partial}(\chi c)=c \bar{\partial} \chi$ extends to a bounded smooth $\bar{\partial}$-closed $(0,1)$-form in $\Omega$. Let $T_{\Omega}$ be a linear solution operator for the $\bar{\partial}$-equation in $\Omega$ (i.e., $\bar{\partial}\left(T_{\Omega} \alpha\right)=\alpha$ for each $\bar{\partial}$-closed smooth $(0,1)$-form $\alpha$ on $\Omega$ ) which is bounded in the sup norm (and also in any $\mathcal{C}^{k}$ norm); see [HL1], p. 82 or [Hö1]. The linear operators

$$
\mathcal{A} c=\chi c-T_{\Omega}(c \bar{\partial} \chi), \quad \mathcal{B} c=(\chi-1) c-T_{\Omega}(c \bar{\partial} \chi)
$$

then satisfy the required properties with respect to the neighborhoods $A^{\prime}, B^{\prime}, C^{\prime}$. In fact, since $T_{\Omega}$ is bounded in all $\mathcal{C}^{k}$ norms, so are the operators $\mathcal{A}$ and $\mathcal{B}$.

Remarks. 1. Gromov requires the existence of decompositions (2.1) with the sup norm estimates on $\mathcal{A}_{j}(c)$ and $\mathcal{B}_{j}(c)$ only depending on $A$ and $B$ and not on the neighborhoods (see sect. 1.5.A in [Gro]). The proof of lemma 2.4 shows that this is possible if we can solve the $\bar{\partial}$-equation with the sup norm estimates on a system of neighborhoods of $A \cup B$ so that the constant in these estimates only depends on $A$ and $B$. This can be achieved only rarely, for instance when $A \cup B$ is itself a strongly pseudoconvex domain. This problem causes difficulties in sect. 2.7 of [Gro] when crossing a critical point. The remark 1.5.A' in [Gro] is incorrect, with immediate counterexamples. (The union of two holomorphically convex sets in $X$ need not have a basis of Stein neighborhoods, and there may be no decomposition (2.1).)

2. Instead of the Banach space $H^{\infty}$ we could as well use in lemma 2.4 any of the spaces $A^{k}$ (holomorphic functions which are smooth of class $\mathcal{C}^{k}$ up to the boundary) as in [HL3]. The solution operator $T_{\Omega}$ on any strongly pseudoconvex domain is bounded in any of these norms.

The inductive construction of sections of $Z \rightarrow X$ is based on the following geometric result from [HL3].

2.5 Theorem. Let $X$ be a complex manifold and let $A^{\prime} \subset X$ be a strongly pseudoconvex extension of a subset $A \subset A^{\prime}$ (def. 2.1 (ii)). Then there exist pseudoconvex bumps $\left(A_{j}, B_{j}\right)$ in $X, 0 \leq j \leq k$, such that

$$
A=A_{0}, \quad A_{j+1}=A_{j} \cup B_{j} \text { for } 0 \leq j \leq k-1, \quad \text { and } A^{\prime}=A_{k} .
$$


Moreover for any open covering $\left\{U_{i}\right\}$ of $X$ we can choose the bumps such that each $B_{j}$ is contained in some $U_{i}$. If $A^{\prime}$ is a non-critical strongly pseudoconvex extension of $A$ then we may choose each $B_{j}$ to be a convex bump on $A_{j}$.

The following is the main technical result of this paper. It allows us to extend a holomorphic section of $Z \rightarrow X$ from a neighborhood of $A$ to a neighborhood of $A \cup B$ for each pseudoconvex bump $(A, B)$ in $X$, with approximation on $A$.

2.6 Theorem. Let $h: Z \rightarrow X$ be a holomorphic submersion onto a Stein manifold $X$ and let $(A, B)$ be a pseudoconvex bump in $X$. Assume that there is an open neighborhood $\tilde{B} \subset X$ of $B$ such that $Z \mid \tilde{B}=h^{-1}(\tilde{B})$ is isomorphic to a trivial bundle $\tilde{B} \times F$, where $F$ admits a spray. Let $Y$ be a compact Hausdorff space (the parameter space), $Y_{0} \subset Y$ a compact subset, and $Y^{\prime} \subset Y_{0}$ an open set containing $Y_{0}$. Let $U \subset X$ be a neighborhood of $A$. Suppose that $a: X \times Y \rightarrow Z$ is a continuous map such that for each $y \in Y$, $a(\cdot, y): X \rightarrow Z$ is a section of $h: Z \rightarrow X$ which is holomorphic in $U$, and the sections $a_{y}$ for $y \in Y^{\prime}$ are holomorphic on $X$. Then for any metric $d$ on $Z$ and for any $\epsilon>0$ there exists a homotopy $a_{t}: X \times Y \rightarrow Z(0 \leq t \leq 1)$ satisfying

(i) $a_{t}(\cdot, y): X \rightarrow Z$ is a section of $h: Z \rightarrow X$ for each $y \in Y$ and $0 \leq t \leq 1$,

(ii) $a_{0}=a$,

(iii) each section $a_{1}(\cdot, y)$ is holomorphic in a neighborhood of $A \cup B$ (independent of $y$ ),

(iv) the homotopy is fixed for $y$ in a neighborhood of $Y_{0}$, i.e., $a_{t}(\cdot, y)=a(\cdot, y)$ for $y$ near $Y_{0}$ and $0 \leq t \leq 1$, and

(v) $d\left(a_{t}(x, y), a(x, y)\right)<\epsilon$ for $x \in A, y \in Y$ and $0 \leq t \leq 1$.

Theorem 2.6 is proved in sect. 6 below. In the rest of this section we assume that theorem 2.6 holds and prove theorem 1.4. For simplicity we write the proof in the case without the parameter $y$; the proof of the general case is the same.

Proof of theorem 1.4. We may assume that $d$ is complete metric on $Z$. Fix an open covering $\mathcal{U}=\left\{U_{i}\right\}$ of $X$ such that $Z \mid U_{i}$ is a trivial bundle with fiber $F$ for each $i$. Since $X$ is Stein and $K$ is holomorphically convex in $X$, there is a smooth strongly plurisubharmonic exhaustion function $\rho: X \rightarrow \mathbf{R}$ such that $\rho<0$ on $K, 0$ is a regular value of $\rho$, and the given section $f_{0}$ is holomorphic in a neighborhood of $A_{0}=\{x \in X: \rho(x) \leq 0\}$. By theorem 2.5 there is a sequence of compact strongly pseudoconvex domains $A_{0} \subset A_{1} \subset A_{2} \subset \ldots \subset$ $\bigcup_{k=0}^{\infty} A_{k}=X$ such that $A_{k+1}=A_{k} \cup B_{k}$ for each $k$, where $\left(A_{k}, B_{k}\right)$ is a pseudoconvex bump and $B_{k} \subset U_{i}$ for some $i$ (so that $Z$ is trivial over a neighborhood of $B_{k}$ ).

Applying theorem 2.6 to each pseudoconvex bump $\left(A_{k}, B_{k}\right)$ we can inductively construct a sequence of continuous sections $f_{k}: X \rightarrow Z$ (with $f_{0}$ being the given initial section) and homotopies of continuous sections $H_{t}^{k}: X \rightarrow Z(0 \leq t \leq 1)$ satisfying the following properties for each $k=0,1,2, \ldots$ and $0 \leq t \leq 1$ :

(i) $f_{k}$ and $H_{t}^{k}$ are holomorphic in a neighborhood of $A_{k}$;

(ii) $H_{0}^{k}=f_{k}, H_{1}^{k}=f_{k+1}$;

(iii) $d\left(H_{t}^{k}(x), f_{k}(x)\right)<\epsilon / 2^{k+1}$ for all $x \in A_{k}$ and $0 \leq t \leq 1$. 
We begin by applying theorem 2.6 to the data $a=f_{0}, A=A_{0}, B=B_{0}$, and $\epsilon$ replaced by $\epsilon / 2$ to get a homotopy $H_{t}^{0}$ such that the section $H_{1}^{0}=f_{1}$ is holomorphic in a neighborhood of $A_{0} \cup B_{0}=A_{1}$ and the properties (i)-(iii) hold with $k=0$.

Suppose inductively that $k>0$ and we have already constructed sections $f_{l}$ and $H_{t}^{l}$ for $l<k$ and $0 \leq t \leq 1$. Then we take $f_{k}=H_{1}^{k-1}$ (this section is holomorphic in a neighborhood of $A_{k}$ ) and apply theorem 2.6 to the data $a=f_{k}, A=A_{k}, B=B_{k}$, and $\epsilon$ replaced by $\epsilon / 2^{k+1}$, to get $H_{t}^{k}$ and $f_{k+1}=H_{1}^{k}$ satisfying (i)-(iii). This completes the induction step.

Property (iii) with $t=1$ implies for all $k=0,1,2, \ldots$

$$
d\left(f_{k+1}(x), f_{k}(x)\right)<\epsilon / 2^{k+1} \quad\left(x \in A_{k}\right) .
$$

Since the metric $d$ is complete, it follows that the limit $f=\lim _{k \rightarrow \infty} f_{k}: X \rightarrow Z$ exists uniformly on compacts in $X$, it is holomorphic on $X$ since $f_{k}$ is holomorphic on $A_{l}$ for each $k \geq l$, and it satisfies the estimate $d\left(f(x), f_{0}(x)\right)<\epsilon$ for $x \in A_{0}$.

To construct a homotopy $H_{t}$ between $H_{0}=f_{0}$ and $H_{1}=f$ we divide the $t$-interval $[0,1)$ into subintervals $I_{k}=\left[1-2^{-k}, 1-2^{-k-1}\right](k=0,1,2, \ldots)$ and take $H_{t}$ for $t \in I_{k}$ to be the homotopy $H_{t}^{k}$, suitably rescaled. To be precise, let $\lambda_{k}: I_{k} \rightarrow[0,1]$ be the linear bijection $\lambda_{k}(t)=2^{k+1}\left(t-1+2^{-k}\right)$. For each $k=0,1,2, \ldots$ we set

$$
H_{t}(x)=H_{\lambda_{k}(t)}^{k}(x) \quad\left(t \in I_{k}, x \in X\right) .
$$

Clearly this defines a homotopy $H_{t}: X \rightarrow Z$ for $0 \leq t<1$. The property (iii) above implies that $\lim _{t \rightarrow 1} H_{t}=f$ uniformly on compacts in $X$, so by setting $H_{1}=f$ we obtain the required homotopy from $f_{0}$ to $f$. This completes the proof of theorem 1.4, provided that theorem 2.6 holds.

\section{\&3. Holomorphic submersions with sprays.}

The reference for this section are sections 1.1-1.3 in [Gro]. We fist recall the notion of a (fiber dominating) spray associated to a holomorphic submersion $h: Z \rightarrow X$. We also recall the notion of an iterated spray bundle and we prove results on lifting homotopies to iterated spray bundles. This is used in sect. 4 in the proof of h-Runge theorems. While it would be possible to avoid iterated spray bundles, their use simplifies proof of theorem 4.2 .

Let $h: Z \rightarrow X$ be a holomorphic submersion between complex manifolds (not necessarily Stein). For $x \in X$ we denote by $Z_{x}=h^{-1}(x) \subset Z$ the fiber over $x$. At each point $z \in Z$ the tangent space $T_{z} Z$ contains a well defined vertical tangent space

$$
V T_{z}(Z)=\left\{e \in T_{z} Z: D h(z) e=0\right\}=T_{z} Z_{h(z)} .
$$

We denote by $V T(Z)$ the corresponding vertical tangent bundle to $Z$ which is a holomorphic subbundle of the tangent bundle $T Z$. Since $Z$ is not assumed to be Stein, there is in general no splitting of $T Z$ into a direct sum $V T(Z) \oplus E^{\prime}$ for some holomorphic vector 
bundle $E^{\prime} \mapsto Z$. However such a splitting exists over any open Stein subset (or a Stein submanifold) $V \subset Z$ (see [GRo], p.256). Also if $f: X \rightarrow Z$ is a holomorphic section then along the graph $f(X) \subset Z$ the tangent bundle has a canonical splitting

$$
T Z|f(X)=V T(Z)| f(X) \oplus T f(X)
$$

If $p: E \rightarrow Z$ is a holomorphic vector bundle over $Z$, we denote by $E_{z}=p^{-1}(z) \subset E$ its fiber over $z \in Z$ and by $0_{z} \in E_{z}$ the zero element of $E_{z}$.

3.1 Definition. A spray on $Z$ associated to the submersion $h: Z \rightarrow X$ (or a fiber-spray) is a tripple $(E, p, s)$, where $p: E \rightarrow Z$ is a holomorphic vector bundle and $s: E \rightarrow Z$ is a holomorphic map such that for each $z \in Z$ we have

(i) $s\left(E_{z}\right) \subset Z_{h(z)}$ (equivalently, $h \circ p=h \circ s$ ),

(ii) $s\left(0_{z}\right)=z$, and

(iii) the derivative $D s\left(0_{z}\right): T_{0_{z}} E \rightarrow V T_{z}(Z)$ maps the subspace $E_{z} \subset T_{0_{z}} E$ surjectively onto $V T_{z}(Z)$.

We denote the restriction in (iii) by

$$
V D s(z)=D s\left(0_{z}\right) \mid E_{z}: E_{z} \rightarrow V T_{z}(Z)
$$

and call it the vertical derivative of $s$ at the point $0_{z} \in E$. Gromov [Gro] calls such a map $s$ a fiberwise dominating spray, the word dominating referring to the property (iii). We shall call it simply a spray when there is no danger of confusion with def. 1.2, or a fiber-spray if we wish to emphasize the difference between the two notions.

Example. Each spray $s: E \rightarrow F$ in the sense of definition 1.2 induces a fiber-spray $(\tilde{E}, \tilde{p}, \tilde{s})$ associated to the trivial fibration $h: Z=X \times F \rightarrow X$ by taking

$$
\tilde{E}=X \times E, \quad \tilde{p}(x, e)=(x, p(e)) \in X \times F=Z, \quad \tilde{s}(x, e)=(x, s(e)) \in Z .
$$

Hence if $Z \mapsto X$ is a locally trivial bundle whose fiber admits a spray, then $X$ can be covered by open sets $U_{i}$ such that each restriction $Z \mid U_{i}$ admits a (fiber-) spray (but in general there is no global spray over $Z$ ).

The main use of sprays is to lift homotopies of sections of $h: Z \rightarrow X$ to homotopies of sections of a certain vector bundles, thereby linearizing the approximation and gluing problems for such sections. The first result in this direction is

3.2 Lemma. (Gromov [Gro], sec. 1.2.) Let $X$ be a Stein manifold and $h: Z \rightarrow X$ a holomorphic submersion which admits a spray $(E, p, s)$. Then for each holomorphic section $f: X \rightarrow Z$ there exists a holomorphic vector subbundle $E^{\prime}$ of the restricted bundle $E \mid f(X)$ such that $s: E^{\prime} \rightarrow Z$ maps a neighborhood of the zero section in $E^{\prime}$ biholomorphically onto a neighborhood of $f(X)$ in $Z$. In particular, if $f_{t}: X \rightarrow Z(0 \leq t \leq 1)$ is a homotopy of holomorphic sections, then for each $t_{0} \in[0,1]$ and each open relatively compact subset $V \subset \subset X$ there is neighborhood $I_{0} \subset[0,1]$ of $t_{0}$ and a homotopy of holomorphic sections 
$\xi_{t}\left(t \in I_{0}\right)$ of $E^{\prime} \subset E \mid f_{t_{0}}(X)$ over the set $f_{t_{0}}(V)$ such that $\xi_{t_{0}}$ is the zero section and $s \circ \xi_{t}(z)=f_{t}(h(z))$ for $t \in I_{0}$ and $z \in f_{t_{0}}(V)$.

Proof. By definition of the spray the map $s: E \mid f(X) \rightarrow Z$ is the identity on the zero section (which we identify with $f(X) \subset Z$ ) and it is a submersion near the zero section. Denote by $E_{0}=\operatorname{ker} V D s \subset E$ the kernel of the vertical derivative $(3.2)$ and let $\tilde{E}=E / E_{0}$ be the quotient bundle with the quotient projection $\pi: E \rightarrow \tilde{E}$. Since $X$ is Stein, this projection splits over $f(X)$, i.e., there is a holomorphic vector bundle homomorphism $G: \tilde{E}|f(X) \rightarrow E| f(X)$ such that $\pi \circ G$ is the identity on $\tilde{E} \mid f(X)$. If we denote by $E^{\prime}$ the image of $G$, we have a direct sum decomposition

$$
E\left|f(X)=E_{0}\right| f(X) \oplus E^{\prime} .
$$

The restriction $s \mid E^{\prime}: E^{\prime} \rightarrow Z$ maps the zero section of $E^{\prime}$ onto $f(X)$ and its derivative is an isomorphism at each point of the zero section. Hence $s \mid E^{\prime}$ is biholomorphic near the zero section. The second statement follows immediately from this.

Lemma 3.2 allows us to lift short pieces of a homotopy of sections of $Z$ to a homotopy of sections of a vector bundle. In order to lift the entire homotopy we recall from [Gro] the concept of composed and iterated sprays.

3.3 Definition. (Gromov [Gro], sec. 1.3.) (a) Let $\left(E_{1}, p_{1}, s_{1}\right)$ and $\left(E_{2}, p_{2}, s_{2}\right)$ be sprays on $Z$ associated to a submersion $h: Z \rightarrow X$. The composed spray $\left(E^{*}, p^{*}, s^{*}\right)$ over $Z$ is defined by

$$
\begin{gathered}
E^{*}=\left\{\left(e_{1}, e_{2}\right) \in E_{1} \times E_{2}: \quad s_{1}\left(e_{1}\right)=p_{2}\left(e_{2}\right)\right\}, \\
p^{*}\left(e_{1}, e_{2}\right)=p_{1}\left(e_{1}\right), \quad s^{*}\left(e_{1}, e_{2}\right)=s_{2}\left(e_{2}\right) .
\end{gathered}
$$

(b) Let $(E, p, s)$ be a spray on $Z$ associated to $h: Z \rightarrow X$. For each integer $k=1,2,3, \ldots$ the $k$-th iterated spray $\left(E^{(k)}, p^{(k)}, s^{(k)}\right)$ is defined by

$$
\begin{gathered}
E^{(k)}=\left\{e=\left(e_{1}, e_{2}, \ldots, e_{k}\right): e_{j} \in E \text { for } j=1,2, \ldots, k,\right. \\
\left.s\left(e_{j}\right)=p\left(e_{j+1}\right) \text { for } j=1,2, \ldots, k-1\right\}, \\
p^{(k)}(e)=p\left(e_{1}\right), \quad s^{(k)}(e)=s\left(e_{k}\right) .
\end{gathered}
$$

Note that the composed spray is not a spray over $Z$ in the sense of def. 3.1 because $E^{*}$ does not have a natural structure of a holomorphic vector bundle over $Z$ with respect to the projection $p^{*}: E^{*} \rightarrow Z$ (the other requirements are satisfied). In fact $E^{*}$ is the pullback of the vector bundle $p_{2}: E_{2} \rightarrow Z$ by the spray map $s_{1}: E_{1} \rightarrow Z$, so it is a holomorphic vector bundle over $E_{1}$ with the projection $\left(e_{1}, e_{2}\right) \mapsto e_{1}$. Similarly we can define the iterated sprays inductively as the composition of $k$ copies of $(E, p, s)$. We begin by taking $\left(E^{(1)}, p^{(1)}, s^{(1)}\right)=(E, p, s)$. Suppose that $\left(E^{(k-1)}, p^{(k-1)}, s^{(k-1)}\right)$ has already been defined. Let $q^{(k)}: E^{(k)} \rightarrow E^{(k-1)}$ be the pullback of the bundle $p: E \rightarrow Z$ by the spray map $s^{(k-1)}: E^{(k-1)} \rightarrow Z$. Set $p^{(k)}=p^{(k-1)} \circ q^{(k)}: E^{(k)} \rightarrow Z$ and let $s^{k}: E^{(k)} \rightarrow Z$ be the map induced by $s: E \rightarrow Z$ under the pullback. This gives the next iterate $\left(E^{(k)}, p^{(k)}, s^{(k)}\right)$.

The following lemma implies that the restriction of composed and iterated sprays to Stein subsets of $Z$ admit a holomorphic vector bundle structure. 
3.4 Lemma. Let $Y$ be a Stein manifold and let $p_{1}: E_{1} \rightarrow Y$ resp. $p: E \rightarrow E_{1}$ be holomorphic vector bundles over $Y$ resp. over $E_{1}$. Then $E$ has the structure of a holomorphic vector bundle over $Y$ with respect to the projection $p_{1} \circ p: E \rightarrow Y$. In fact, this bundle is isomorphic to the Whitney sum $E_{1} \oplus(E \mid Y)$, where $E \mid Y$ denotes the restriction of $E$ to the zero section $Y \subset E_{1}$ of $E_{1}$.

3.5 Corollary. (Gromov [Gro], sec. 1.3.A') The restriction of any composed or iterated spray bundle on $Z$ to any Stein subset $U \subset Z$ admits a structure of a holomorphic vector bundle over $U$.

Proof of lemma 3.4. Since $E_{1} \rightarrow Y$ is a holomorphic vector bundle over a Stein manifold $Y$, the total space $E_{1}$ is itself a Stein manifold. Denote the points of $E_{1}$ by $(y, e)$, where $y \in Y$ and $p_{1}(y, e)=y$. Let $h_{t}: E_{1} \rightarrow E_{1}$ for $t \in \mathbf{C}$ be the homotopy $h_{t}(y, e)=(y, t e)$. Consider the family of pull-backs $h_{t}^{*}(E) \rightarrow E_{1}$ of the vector bundle $p: E \rightarrow E_{1}$. It follows from Grauert's theorem [Gr4] (Satz I) that all bundles in the family are holomorphically isomorphic; see [Lei] for an elementary proof. In particular the bundles $h_{1}^{*}(E)$ and $h_{0}^{*}(E)$ are isomorphic. The map $h_{1}$ is the identity on $E_{1}$ whence $h_{1}^{*} E=E$. The map $h_{0}=p_{1}$ is the projection of $E_{1}$ onto the zero section $Y \subset E_{1}$ and hence $h_{0}^{*}(E)=p_{1}^{*}(E)=p_{1}^{*}(E \mid Y)$. The latter bundle is clearly isomorphic to the Whitney sum $E_{1} \oplus(E \mid Y)$ which is a holomorphic vector bundle over $Y$. This proves lemma 3.4 .

The next result indicates the main application of iterated sprays.

3.6 Proposition. Let $f_{t}: X \rightarrow Z(t \in[0,1])$ be a homotopy of holomorphic sections of a holomorphic submersion $h: Z \rightarrow X$. Assume that the base $X$ is Stein and that $h: Z \rightarrow X$ admits a spray $(E, p, s)$. Then for each open relatively compact subset $V \subset \subset X$ there are an integer $k>0$ and a homotopy of holomorphic sections $\xi_{t}(0 \leq t \leq 1)$ of the iterated spray bundle $E^{(k)}$ (3.4) over the set $f_{0}(V) \subset Z$ such that

$$
\xi_{0}(z)=z, \quad s^{(k)}\left(\xi_{t}(z)\right)=f_{t}(h(z)) \quad\left(z \in f_{0}(V), 0 \leq t \leq 1\right) .
$$

Proof. For each fixed $t \in[0,1]$ we can apply lemma 3.2 to lift the sections $f_{\tau}$ for $\tau$ near $t$ by the spray map $s$ to a homotopy of holomorphic sections of $E \mid f_{t}(V)$. Hence by compactness of $[0,1]$ there are numbers $0=t_{0}<t_{1}<t_{2}<\cdots<t_{k}=1$ such that for each $j=0,1, \ldots, k-1$ there exists a homotopy of holomorphic sections $\xi_{t}^{j}$ of $E \mid f_{t_{j}}(V)$ for $t_{j} \leq t \leq t_{j+1}$ satisfying

$$
s \circ \xi_{t}^{j}\left(f_{t_{j}}(x)\right)=f_{t}(x) \quad\left(x \in V, t_{j} \leq t \leq t_{j+1}\right) .
$$

In particular we have $s \circ \xi_{t_{j+1}}^{j}\left(f_{t_{j}}(x)\right)=f_{t_{j+1}}(x)$ for $j=0,1, \ldots, k-1$. Comparing these compatibility conditions with those defining the iterated spray bundle $E^{(k)}(3.4)$ we see that these $k$ families can be joined into a single family of sections $\xi_{t}(0 \leq t \leq 1)$ of $E^{(k)} \mid f_{0}(V)$. Explicitly we define for $x \in V$ and $t_{j} \leq t \leq t_{j+1}$ :

$$
\xi_{t}\left(f_{0}(x)\right)=\left(\xi_{t_{1}}^{0}\left(f_{0}(x)\right), \xi_{t_{2}}^{1}\left(f_{t_{1}}(x)\right), \ldots, \xi_{t_{j}}^{j-1}\left(f_{t_{j-1}}(x)\right), \xi_{t}^{j}\left(f_{t_{j}}(x)\right), 0, \ldots, 0\right) \in E^{(k)}
$$


(the last $k-j-1$ components are the zero elements in the fiber of $E$ over $s \circ \xi_{t}^{j}\left(f_{t_{j}}(x)\right)=$ $\left.f_{t}(x)\right)$. One easily verifies that these sections satisfy the stated conditions.

\section{\&4. The h-Runge theorems.}

In this section we prove Runge-type approximation theorems for holomorphic sections of submersions with a spray over a Stein base. Theorems 4.1 and 4.2 are due to Gromov [Gro], while theorem 4.5 is new. The idea in these proofs is essentially the same as in Grauert's paper [Gr2] where the analogous results had been proved for sections of bundles over Stein spaces whose fiber is a complex Lie group $L$ and the transition functions are Lie group automorphisms of $L$.

To motivate the discussion we recall that, if $X$ is a Stein manifold and $K \subset X$ is a compact holomorphically convex subset, we can approximate each function holomorphic in a neighborhood of $K$ uniformly on $K$ by functions holomorphic on $X$. This is the OkaWeil theorem [Hö2] which extends the classical Runge theorem for planar sets $K \subset \mathbf{C}$ with connected complement. Of course we cannot expect such results for sections of an arbitrary holomorphic submersion over $X$; in particular this fails for maps of Stein manifolds into a hyperbolic complex manifold $F$. On the other hand, if $h: Z \rightarrow X$ admits a fiber-spray (def. 3.1) then the Runge approximation property is homotopy independent in the following sense: If $f_{t}$ is a homotopy of sections in a neighborhood of $K$ such that $f_{0}$ has a holomorphic extension to $X$, then each section in the homotopy can be approximated on $K$ by sections holomorphic on $X$. Following Gromov we call such results $h$-Runge theorems. We first state the special case, theorem 4.1. The general parametric case is explained in theorem 4.2 .

4.1 Theorem. Let $X$ be a Stein manifold and $h: Z \rightarrow X$ a holomorphic submersion which admits a fiber-spray (def. 3.1). Let $K \subset X$ be a compact holomorphically convex set. Assume that $U \subset X$ is an open set containing $K$ and $f_{t}: U \rightarrow Z(0 \leq t \leq 1)$ is a homotopy of holomorphic sections of $h: Z \rightarrow X$ over $U$ such that $f_{0}$ extends to a holomorphic section over $X$. Then for any metric $d$ on $Z$ and any $\epsilon>0$ there exists a continuous family of holomorphic sections $\tilde{f}_{t}: X \rightarrow Z(0 \leq t \leq 1)$ such that $\tilde{f}_{0}=f_{0}$ and

$$
d\left(\tilde{f}_{t}(x), f_{t}(x)\right)<\epsilon \quad(x \in K, 0 \leq t \leq 1) .
$$

Remark. In fact we will prove the following stronger result: There is a continuous family of continuous sections $g_{t, u}: X \rightarrow Z(0 \leq t, u \leq 1)$ which are holomorphic in a neighborhood of $K$ and satisfy:

(a) $g_{t, 0}=f_{t}$ on $K$ for all $0 \leq t \leq 1$,

(b) the section $\tilde{f}_{t}=g_{t, 1}$ is holomorphic on $X$ for each $t \in[0,1]$,

(c) $g_{0, u}=f_{0}$ for all $0 \leq u \leq 1$, and

(d) $d\left(g_{t, u}(x), f_{t}(x)\right)<\epsilon$ for $x \in K$ and $0 \leq t, u \leq 1$.

The existence of such a homotopy $g_{t, u}$ connecting $f_{t}$ and $\tilde{f}_{t}$ comes from the proof of theorem 4.1 and will be useful to us in sect. 6 . 
Proof. Let $(E, p, s)$ be the spray on $Z$ associated to $h$. After shrinking $U$ around $K$ we obtain by proposition 3.6 an integer $k>0$ and a homotopy of sections $\xi_{t}$ over the set $f_{0}(U) \subset Z$ of the iterated spray bundle $p^{(k)}: E^{(k)} \rightarrow Z$ which are mapped back to $f_{t}$ by $s^{(k)}: E^{(k)} \rightarrow Z$. In particular $\xi_{0}$ is the zero section. Since $X$ is Stein, the restriction $E^{(k)} \mid f_{0}(X)$ admits the structure of a holomorphic vector bundle by corollary 3.5 . It now suffices to approximate $\xi_{t}$ on the holomorphically convex subset $f_{0}(K)$ of $f_{0}(X)$ by a homotopy of holomorphic sections of $E^{(k)} \mid f_{0}(X)$ (keeping the zero section fixed) and to take $\tilde{f}_{t}$ to be their images in $Z$ by the spray map $s^{(k)}$. This can be done by the usual Oka-Weil approximation theorem for sections of a holomorphic vector bundle, and it can be reduced to the aproximation of functions by embedding the given bundle as a subbundle of a trivial bundle. Even though this is standard, we outline the proof for later purposes.

For convenience we let $p^{\prime}: E^{\prime}=f_{0}^{*}\left(E^{(k)}\right) \rightarrow X$ be the pull-back of $E^{(k)}$ by the section $f_{0}: X \rightarrow Z$, and we denote by $s^{\prime}: E^{\prime} \rightarrow Z$ the holomorphic map induced by the spray $s^{(k)}: E^{(k)} \rightarrow Z$. We may then consider $\xi_{t}$ as sections of the bundle $p^{\prime}: E^{\prime} \rightarrow X$ over $U \subset X$ such that $\xi_{0}$ is the zero section and $s^{\prime} \circ \xi_{t}(x)=f_{t}(x)$ for all $x \in U$ and $0 \leq t \leq 1$. Choose a smooth function $\chi: X \rightarrow[0,1]$ which is identically one in a neighborhood of $K$ and has compact support contained in $U$. Since $K$ is holomorphically convex in $X$, there is a smooth plurisubharmonic exhaustion function $\rho: X \rightarrow \mathbf{R}_{+}$which vanishes in a neighborhood $U_{0} \subset \subset U$ of $K$ and is strictly positive on the support of $d \chi$. We can choose $\rho$ such that for each fixed value of $\tau>1$ there is a section $v_{t}$ of $E^{\prime}$ which solves the equation

$$
\bar{\partial} v_{t}=\bar{\partial}\left(\chi \xi_{t}\right)=\xi_{t} \bar{\partial} \chi
$$

and whose $L^{2}$ norm with weight $e^{-\tau \rho}$ (measured in a fixed hermitian metric) is bounded on each compact set in $X$ by a constant times the norm of the data $\xi_{t} \bar{\partial} \chi$ (with a constant independent of $\tau$ ). By Hörmander [Hö1] such a solution is given by a linear operator $v_{t}=T_{\tau}\left(\xi_{t} \bar{\partial} \chi\right)$. We have $v_{0}=0$, each $v_{t}$ is smooth, and the family is continuous in $t$. Set

$$
\tilde{g}_{t, u}=\chi \xi_{t}-u v_{t}, \quad g_{t, u}=s^{\prime} \circ \tilde{g}_{t, u} \quad(0 \leq t, u \leq 1)
$$

Clearly $g_{t, u}: X \rightarrow Z$ is a continuous family of sections of $Z \rightarrow X$ which satisfies (a)-(c) in the remark following theorem 4.1. By choosing $\tau$ sufficiently large (depending on $\epsilon$ ) the family $g_{t, u}$ will also satisfy (d) which can be seen as follows. When $\tau \rightarrow \infty$, the $L^{2}$ norm with weight $e^{-\tau \rho}$ of $\xi_{t} \bar{\partial} \chi$ tends to zero since $\rho>0$ on supp $\bar{\partial} \chi$. Since $\rho$ vanishes in $U_{0}$, it follows that unweighted $L^{2}\left(U_{0}\right)$ norm $\left\|v_{t}\right\|_{L^{2}\left(U_{0}\right)}$ tends to zero as $\tau \rightarrow+\infty$. By the Cauchy estimates the sup norm of $v_{t} \mid K$ tends to zero and hence the sections $g_{t, u}$ converge to $f_{t}$ as $\tau \rightarrow+\infty$, uniformly in $t$ and $u$. In particular, $\tilde{f}_{t}=g_{t, 1}(0 \leq t \leq 1)$ is a homotopy of holomorphic sections of $Z \rightarrow X$ satisfying theorem 4.1 .

We will also need the following parametric version of the h-Runge theorem.

4.2 Theorem. Let $X$ be a Stein manifold and $h: Z \rightarrow X$ a holomorphic submersion which admits a spray (def. 3.1). Let $K \subset X$ be a compact holomorphically convex set and let $U, V \subset X$ be open, relatively compact subsets in $X$ such that $K \subset U \subset V$. Let $Y$ be a compact Hausdorff space (the parameter space), $Y_{0} \subset Y$ a compact subset, and $Y^{\prime} \subset Y$ 
an open set containing $Y_{0}$. Assume that $f_{y, t}: U \rightarrow Z$ is a family of holomorphic sections of $h: Z \rightarrow X$, depending continuously on $y \in Y$ and $0 \leq t \leq 1$, such that the sections $f_{y, 0}$ $(y \in Y)$ and $f_{y, t}\left(y \in Y^{\prime}, 0 \leq t \leq 1\right)$ extend to holomorphic sections over $X$. Then for each $\epsilon>0$ there exists a continuous family of holomorphic sections $\tilde{f}_{y, t}: V \rightarrow Z$ over $V$ $(y \in Y, 0 \leq t \leq 1)$ satisfying

(a) $\tilde{f}_{y, 0}=f_{y, 0}$ for all $y \in Y$,

(b) $\tilde{f}_{y, t}=f_{y, t}$ for all $y \in Y_{0}$ and $0 \leq t \leq 1$, and

(c) $d\left(\tilde{f}_{y, t}(x), f_{y, t}(x)\right)<\epsilon$ for all $x \in K, y \in Y$ and $0 \leq t \leq 1$.

Remarks. 1. As in theorem 4.1 the proof will show that the approximating family $\tilde{f}_{y, t}$ can be chosen so that it can be connected to the initial family $f_{y, t}$ by a homotopy of sections $g_{y, t, u}(u \in[0,1])$ such that the homotopy is fixed for each $y \in Y_{0}$ (where $\tilde{f}_{y, t}=f_{y, t}$ ).

2. By a standard limiting procedure we could obtain a family $\tilde{f}_{y, t}$ on all of $X$, but we shall not need this.

Proof. We begin by reducing to the approximation problem for families of sections of an iterated spray bundle. This is essentially proposition 3.6 with the addition of the parameter $y \in Y$. Let $(E, p, s)$ be a spray on $Z$ and $\left(E^{(k)}, p^{(k)}, s^{(k)}\right)$ its $k$-th iterated spray (3.4).

4.3 Proposition. (Assumptions as in theorem 4.2.) Let $U^{\prime}$ be an open set in $X$ such that $K \subset U^{\prime} \subset \subset U$. Then there are an integer $k>0$ and a continuous family of holomorphic sections $\xi_{y, t}$ of $E^{(k)} \mid f_{y, 0}\left(U^{\prime}\right) \quad(y \in Y, 0 \leq t \leq 1)$ such that

(i) $\xi_{y, 0}$ is the zero section for each $y \in Y$,

(ii) $\xi_{y, t}$ extends to a holomorphic section of $E^{(k)} \mid f_{y, 0}(V)$ for each $y$ in a neighborhood of $Y_{0}$ and $t \in[0,1]$, and

(iii) $s^{(k)} \circ \xi_{y, t}\left(f_{y, 0}(x)\right)=f_{y, t}(x)$ for all $x \in U^{\prime}, y \in Y$ and $0 \leq t \leq 1$. Moreover, for each $y$ in a neighborhood of $Y_{0}$ this holds for all $x \in V$.

Proof. It suffices to prove that each fixed $t_{0} \in[0,1]$ has a neighborhood $I_{0} \subset[0,1]$ such that there exists a family of holomorphic sections $\xi_{y, t}$ of the vector bundle $E \mid f_{y, t_{0}}\left(U^{\prime}\right)$, depending continuously on $y \in Y$ and $t \in I_{0}$, such that $\xi_{y, t_{0}}$ is the zero section and

$$
s \circ \xi_{y, t}(z)=f_{y, t}(h(z)) \quad\left(z \in f_{y, t_{0}}\left(U^{\prime}\right), y \in Y, t \in I_{0}\right) .
$$

Moreover, for $y$ in a neighborhood of $Y_{0}$ the property (4.2) must hold over the larger set $z \in f_{y, t_{0}}(V)$. Proposition 4.3 then follows from this as in the proof of proposition 3.6 by using the compactness of $[0,1]$ and combining the finitely many families of sections $\xi_{y, t}^{j}$ obtained over $k$ subintervals $t \in\left[t_{j}, t_{j+1}\right] \subset[0,1]$ into a single family of sections of the iterated spray bundle $E^{(k)}$ over the sets $f_{y, 0}\left(U^{\prime}\right)$ resp. $f_{y, 0}(V)$.

We will consider the case $t_{0}=0$ when all initial sections $f_{y, 0}$ exist over $V$. The only difference for $t_{0}>0$ is that some sections only exist over the smaller set $U^{\prime}$, but the proof goes through in the same way. Denote by $E_{0}=\operatorname{ker} V D s \subset E$ the kernel of the vertical derivative of $s(3.2)$ and let $\pi: E \mapsto \tilde{E}=E / E_{0}$ be the quotient projection. Recall that a 
holomorphic splitting of $\pi$ is a holomorphic vector bundle homomorphism $G: \tilde{E} \rightarrow E$ such that $\pi \circ G$ is the identity on $\tilde{E}$; in such case we have $E=E_{0} \oplus G(\tilde{E})$. A splitting exists over any Stein subset of $Z$ [GRo, p. 256].

4.4 Lemma. (Hypotheses as in theorem 4.2.) There is a family of holomorphic splittings $G_{y}: \tilde{E}\left|f_{y, 0}(V) \rightarrow E\right| f_{y, 0}$ which depends continuously on $y \in Y$, and hence there is a holomorphic direct sum splitting

$$
E\left|f_{y, 0}(V)=E_{0}\right| f_{y, 0}(V) \oplus E_{y}^{\prime}
$$

depending continuously on $y \in Y$.

Remark. We may consider the restricted bundles in lemma 4.4 as subsets of the bundle $E$ resp. $\tilde{E}=E / E_{0}$, and the continuity of the family $G_{y}$ with respect to $y$ should be understood in this sense. Lemma 4.4 also holds if we replace $t=0$ by an arbitrary $t_{0} \in[0,1]$, except that in this case one must replace for each $y \in Y \backslash Y^{\prime}$ the set $V$ in (4.3) by the smaller set $U^{\prime}$ (since the section $f_{y, t_{0}}$ is only defined on $U^{\prime}$ ).

Proof of lemma 4.4. For each fixed $y \in Y$ the section $f_{y, 0}(X) \subset Z$ (which is a Stein submanifold of $Z$ ) is contained in an open Stein set $D_{y} \subset Z$ according to a theorem of Siu [Siu]. Hence by compactness of $Y$ there is an open covering $\left\{Y_{j}: 1 \leq j \leq J\right\}$ of $Y$ and a family of open Stein subsets $\left\{D_{j}: 1 \leq j \leq J\right\}$ of $Z$ such that $f_{y, 0}(V) \subset D_{j}$ when $y \in Y_{j}$. Let $\chi_{j}$ be a continuous partition of unity on $Y$ subordinate to the covering $\left\{Y_{j}\right\}$. Let $H_{j}: \tilde{E}\left|D_{j} \rightarrow E\right| D_{j}$ be a splitting of $\pi: E \rightarrow \tilde{E}$ over $D_{j}$ (such $H_{j}$ exists since $D_{j}$ is Stein). Then the family

$$
G_{y}=\sum_{j=1}^{J} \chi_{j}(y) H_{j}: \quad \tilde{E}\left|f_{y, 0}(V) \rightarrow E\right| f_{y, 0}(V)
$$

satisfies lemma 4.4. Note that the map $G_{y}$ is well defined since the coefficient $\chi_{j}(y)$ vanishes when $f_{y, 0}(V)$ is not contained in $D_{j}$, and $G_{y}$ is a splitting of $\pi$ since it is a convex linear combination of finitely many splittings.

We continue with the proof of proposition 4.3. By the inverse function theorem the map $s: E_{y}^{\prime} \rightarrow Z$ (the restriction of the spray map $s: E \rightarrow Z$ to $E_{y}^{\prime}$ ) is a biholomorphic map from a neighborhood of the zero section of $E_{y}^{\prime}$ onto a neighborhood of the set $f_{y, 0}(V)$ in $Z$ (since the vertical derivative $V D s$ is an isomorphism when restricted to $E_{y}^{\prime}$ ). Denote the local inverse of this map by $u_{y}$. Because of continuous dependence on the data on $y \in Y$ and the compactness of $Y$ the neighborhoods on which the maps $u_{y}$ are defined can be chosen uniformly with respect to $y \in Y$. Hence there is a $t_{1}>0$ such that for $0 \leq t \leq t_{1}$ and $y \in Y$ the set $f_{y, t}\left(U^{\prime}\right)$ belongs to the domain of $u_{y}$. The sections $\xi_{y, t}=u_{y} \circ f_{y, t} \circ h: f_{y, 0}\left(U^{\prime}\right) \rightarrow E_{y}^{\prime} \subset E$ for $0 \leq t \leq t_{1}$ then satisfy (4.2). This completes the proof of proposition 4.3 .

To complete the proof of theorem 4.2 it remains to approximate the sections $\xi_{y, t}$ uniformly on $f_{y, 0}(K)$ by holomorphic sections of $E^{(k)} \mid f_{y, 0}(V)$. We shall first do this locally with respect to $y$ and finally patch the approximations together. Let $Y^{\prime} \supset Y_{0}$ be 
a neighborhood of $Y_{0}$ such that the sections $\xi_{y, t}$ for $y \in Y^{\prime}$ are defined and holomorphic over the set $f_{y, 0}(V) \subset Z$. As in the proof of lemma 4.4 there exist a finite open covering $\left\{Y_{j}: 1 \leq j \leq J\right\}$ of $Y$ and open Stein subsets $D_{j} \subset Z$ such that $f_{y, 0}(V) \subset D_{j}$ for all $y$ in a neighborhood $Y_{j}^{\prime} \supset Y_{j}$ of $\bar{Y}_{j}$. By corollary 3.5 the restriction $E^{(k)} \mid D_{j}$ has a vector bundle structure and we can embed it as a vector subbundle of a trivial bundle $D_{j} \times \mathbf{C}^{N}$. With this identification we may write

$$
\xi_{y, t}\left(f_{y, 0}(x)\right)=\left(f_{y, 0}(x), g_{y, t}(x)\right)
$$

where $g_{y, t}: U^{\prime} \rightarrow \mathbf{C}^{N}$ is a holomorphic map. Note that $g_{y, 0}=0$ for all $y \in \bar{Y}_{j}$, and $g_{y, t}$ extends holomorphically to $V$ for $y \in Y^{\prime} \cap Y_{j}^{\prime}$. Choose a continuous function $u: Y \rightarrow[0,1]$ which vanishes in smaller neighborhood of $Y_{0}$ and is identically one outside $Y^{\prime}$. In now suffices to approximate the functions $g_{y, t}$ by functions holomorphic in $V$ without changing their values for $y \in Y^{\prime}$ (when they are already holomorphic in $V$ ). This is done by taking

$$
\tilde{g}_{y, t}(x)=u(y) \chi(x) g_{y, t}(x)-v_{y, t}(x)
$$

where $v_{y, t}$ is the solution of the $\bar{\partial}$-equation

$$
\bar{\partial}_{x} v_{y, t}(x)=u(y) g_{y, t}(x) \bar{\partial}_{x} \chi(x)
$$

with minimal $L^{2}$-norm with respect to a suitable weight and $\chi: X \rightarrow \mathbf{R}_{+}$is a cut-off function as in the proof of theorem 4.1. Since $u(y)=0$ for $y$ near $Y_{0}$, we have $v_{y, t}=0$ and hence $\tilde{g}_{y, t}=g_{y, t}$ for such $y$.

This gives for each $j=1,2, \ldots, J$ an approximating family of holomorphic sections $\xi_{y, t}^{j}$ of $E^{(k)} \mid f_{y, 0}(V)$ for $y$ in a neighborhood $Y_{j}^{\prime}$ of $\bar{Y}_{j}$. To conclude the proof of theorem 4.2 it suffices to combine these families into a single family of sections $\tilde{\xi}_{y, t}(y \in Y)$ and then take

$$
\tilde{f}_{y, t}=s^{(k)} \circ \tilde{\xi}_{y, t} \circ f_{y, 0} \quad(y \in Y, 0 \leq t \leq 1) .
$$

However we cannot do the patching by the usual partition of unity in the $y$ variable since the vector bundle structures on different restrictions $E^{(k)} \mid D_{j}$ may not agree on their intersection. Instead we can do a stepwise extension as follows. For $y \in Y_{1}^{\prime}$ we take $\tilde{\xi}_{y, t}=\xi_{y, t}^{1}$. Choose a continuous function $\chi_{1}: Y \rightarrow[0,1]$ such that $\chi_{1}(y)=1$ for $y \in \bar{Y}_{1}$ and supp $\chi_{1} \subset Y_{1}^{\prime}$. For $y \in Y_{2}^{\prime}$ we then set

$$
\tilde{\xi}_{y, t}=\chi_{1}(y) \xi_{y, t}^{1}+\left(1-\chi_{1}(y)\right) \xi_{y, t}^{2}
$$

where the linear combination is taken with respect to a vector bundle structure on $E^{(k)} \mid D_{2}$. This definition is good since $\chi_{1}(y)=0$ for those values of $y$ (i.e., for $y \in Y \backslash Y_{1}^{\prime}$ ) for which the section $\xi_{y, t}^{1}$ is not defined, and so the patching only occurs over the set $y \in Y_{2}^{\prime}$. Clearly the family (4.5) extends continuously to all parameter values $y \in Y_{1}^{\prime} \cup Y_{2}^{\prime}$ and it equals the previously chosen family of sections for $y \in Y_{1}$. Moreover, for $y \in Y_{1}^{\prime} \cap Y_{2}^{\prime}$ both sections $\xi_{y, t}^{1}$ and $\xi_{y, t}^{2}$ approximate the initial section $\xi_{y, t}$ over $f_{y, 0}(K)$, and hence the same is true for their convex combination (4.5). We now continue in the same way by patching the family 
(4.5) with $\xi_{y, t}^{3}$ over the parameter set $y \in Y_{3}^{\prime}$ with respect to the vector bundle structure on $E^{(k)} \mid D_{3}$. In finite number in steps we obtain a continuous family of sections $\tilde{\xi}_{y, t}$ such that the sections (4.4) satisfy theorem 4.2 .

Our next result, which is an immediate application of theorem 4.2, is essential in our approach to extending a holomorphic section across a pseudoconvex bump in the critical case.

4.5 Theorem. Let $X$ be a Stein manifold and $Z=X \times F \rightarrow X$ a trivial bundle whose fiber $F$ admits a spray (def. 1.2). Suppose that $U \subset X$ is a Stein domain which is Runge in $X$ and $\Theta_{u}: X \rightarrow X$ is a family of holomorphic mappings, depending continuously on $u \in[0,1]$, such that

(i) $\Theta_{0}$ is the identity on $X$,

(ii) $\Theta_{u}(U) \subset U$ for all $u \in[0,1]$, and

(iii) $\Theta_{1}(X) \subset U$.

Let $f_{t}: U \rightarrow Z(0 \leq t \leq 1)$ be a homotopy of holomorphic sections such that $f_{0}$ and $f_{1}$ extend holomorphically to $X$. Let $d$ be any metric on $Z$. For each choice of a compact set $K \subset U$, a relatively compact set $V \subset \subset X$, and $\epsilon>0$ there exists a homotopy of holomorphic sections $\tilde{f}_{t}: V \rightarrow Z(0 \leq t \leq 1)$ such that $\tilde{f}_{0}=f_{0}, \tilde{f}_{1}=f_{1}$, and

$$
d\left(\tilde{f}_{t}(x), f_{t}(x)\right)<\epsilon \quad(x \in K, 0 \leq t \leq 1) .
$$

Remarks. 1. In a typical application of theorem 4.5 the sets $U \subset X$ are bounded convex domains in $\mathbf{C}^{n}$ and $\Theta_{u}$ is a family of linear contractions to a point in $U$. By a limiting argument it is possible to prove that such a holomorphic homotopy $\tilde{f}_{t}$ exists on all of $X$, but we shall not need this.

2. The analogous result holds, with the same proof, for parametrized families of sections. We leave out the obvious details.

Proof. By reparametrizing the family $f_{t}$ we may assume that for some small $\delta>0$ we have $f_{t}=f_{0}$ for $0 \leq t \leq \delta$ and $f_{t}=f_{1}$ for $1-\delta \leq t \leq 1$. Choose a continuous function $u:[0,1] \rightarrow[0,1]$ such that $u(t)=0$ for $t$ near 0 or 1 , and $u(t)=1$ for $\delta \leq t \leq 1-\delta$. We shall identify sections of the trivial bundle $X \times F \rightarrow X$ with mappings $X \rightarrow F$. Set

$$
f_{t, s}=f_{t} \circ \Theta_{(1-s) u(t)} \quad(0 \leq t, s \leq 1) .
$$

This family satisfies the following properties:

(a) $f_{t, s}$ is defined and holomorphic in $U$ for each $0 \leq t, s \leq 1$,

(b) $f_{t, 0}=f_{t} \circ \Theta_{u(t)}$ is defined and holomorphic on all of $X$ for each $t \in[0,1]$,

(c) $f_{t, 1}=f_{t} \circ \Theta_{0}=f_{t}$ for each $t \in[0,1]$, and

(d) $f_{0, s}=f_{0} \circ \Theta_{u(0)}=f_{0}$ and $f_{1, s}=f_{1} \circ \Theta_{u(1)}=f_{1}$ for all $s \in[0,1]$.

It remains to apply theorem 4.2 with the parameter space $t \in Y=[0,1]$ and the subspace $Y_{0}=\{0,1\}$. (Note that our current variable $s$ plays the role of the time parameter $t$ 
in theorem 4.2.) If $\tilde{f}_{t, s}$ is the approximating family of holomorphic sections in $V$ as in theorem 4.2 then the sections $\tilde{f}_{t}=\tilde{f}_{t, 1}(0 \leq t \leq 1)$ satisfy theorem 4.5 .

\section{\&5. Gluing holomorphic sections over Cartan pairs.}

The main results of this section are theorems 5.1 and 5.5 on gluing holomorphic sections over Cartan pairs. This can be found in sect. 1.6 of [Gro] and is similar to the corresponding results of Grauert [Gr3] and Cartan [Car].

5.1 Theorem. Let $h: Z \rightarrow X$ be a holomorphic submersion onto a Stein manifold $X$. Let $d$ be a metric on $Z$. Let $(A, B)$ be a Cartan pair in $X$ (def. 2.3) such that the set $C=A \cap B$ is Runge in $B$. Suppose that $\tilde{B}$ is an open neighborhood of $B$ in $X$ such that the restriction $Z \mid \tilde{B}=h^{-1}(\tilde{B})$ admits a fiber-spray (def. 3.1). Let $\tilde{A} \subset X$ be an open neighborhood of $A$ and $a: \tilde{A} \rightarrow Z$ a holomorphic section of $Z \rightarrow X$ over $\tilde{A}$. Then for each $\epsilon>0$ there is a $\delta>0$ satisfying the following property. If $b: \tilde{B} \rightarrow Z$ is a holomorphic section satisfying $d(a(x), b(x))<\delta$ for $x \in \tilde{C}=\tilde{A} \cap \tilde{B}$, there exist homotopies $a_{t}$ (resp. $b_{t}$ ), $0 \leq t \leq 1$, of holomorphic sections over a neighborhood $A^{\prime}$ of $A$ (resp. over a neighborhood $B^{\prime}$ of $B$ ) such that $a_{0}=a, b_{0}=b, a_{1}=b_{1}$ on $C^{\prime}=A^{\prime} \cap B^{\prime}$, and

$$
\begin{array}{ll}
d\left(a_{t}(x), a(x)\right)<\epsilon & \left(x \in A^{\prime}, 0 \leq t \leq 1\right) \\
d\left(b_{t}(x), b(x)\right)<\epsilon & \left(x \in C^{\prime}, 0 \leq t \leq 1\right) .
\end{array}
$$

Using sprays we shall reduce the proof of theorem 5.1 to the model case described by the following proposition; this is analogous to the classical Cartan-Grauert attaching lemma [Gr3], [Car]. We denote by $H^{\infty}\left(\Omega, \mathbf{C}^{n}\right)$ the Banach space of bounded holomorphic maps $\Omega \rightarrow \mathbf{C}^{n}$ equipped with the sup norm over all components.

5.2 Proposition. Let $(A, B)$ be a Cartan pair in a Stein manifold $X$ such that $C=A \cap B$ is Runge in $B$. Let $\tilde{C} \subset X$ be an open neighborhood of $C, U \subset \mathbf{C}^{n}$ an open neighborhood of the origin in $\mathbf{C}^{n}$, and $\psi_{0}: \tilde{C} \times U \rightarrow \mathbf{C}^{n}$ a bounded holomorphic map such that for each $x \in \tilde{C}, \psi_{0}(x, 0)=0$ and $\psi_{0}(x, \cdot): U \rightarrow \mathbf{C}^{n}$ is injective (i.e., biholomorphic onto its image). Then there are neighborhoods $A^{\prime} \supset A$ and $B^{\prime} \supset B$ with $C^{\prime}=A^{\prime} \cap B^{\prime} \subset \subset \tilde{C}$, a neighborhood $W$ of $\psi_{0}$ in the Banach space $H^{\infty}\left(\tilde{C} \times U, \mathbf{C}^{n}\right)$, and smooth Banach space operators $\mathcal{A}^{\prime}: W \rightarrow H^{\infty}\left(A^{\prime}, \mathbf{C}^{n}\right), \mathcal{B}^{\prime}: W \rightarrow H^{\infty}\left(B^{\prime}, \mathbf{C}^{n}\right)$, with $\mathcal{A}^{\prime}\left(\psi_{0}\right)=0$ and $\mathcal{B}^{\prime}\left(\psi_{0}\right)=0$, such that for each $\psi \in W$ the bounded holomorphic maps $\alpha=\mathcal{A}^{\prime}(\psi): A^{\prime} \rightarrow \mathbf{C}^{n}, \beta=$ $\mathcal{B}^{\prime}(\psi): B^{\prime} \rightarrow \mathbf{C}^{n}$ satisfy

$$
\psi(x, \alpha(x))=\beta(x) \quad\left(x \in A^{\prime} \cap B^{\prime}\right) .
$$

Moreover, if $\psi \in W$ satisfies $\psi(x, 0)=0$ for $x \in \tilde{C}$ then $\mathcal{A}^{\prime}(\psi)=0$ and $\mathcal{B}^{\prime}(\psi)=0$.

Remark. We can view a pair of maps satisfying (5.1) as a section of a nonlinear bundle over $A^{\prime} \cup B^{\prime}$ obtained by patching the trivial bundles over $A^{\prime}$ resp. $B^{\prime}$ by the map $\psi$. For later application to parametrized families it is convenient to have a canonically given solution (i.e., by operators), although this could be avoided by a suitable analogue of Satz 8 in $[\mathrm{Gr} 2]$. 
Proof of proposition 5.2. By shrinking $\tilde{C}$ we may assume that it is Runge in a neighborhood $B_{0}$ of $B$. We choose neighborhoods $A^{\prime} \supset A$ and $B^{\prime} \supset B$ as in lemma 2.4 so that $B^{\prime} \subset B_{0}, C^{\prime}=A^{\prime} \cap B^{\prime} \subset \subset \tilde{C}$, and there are bounded linear operators $\mathcal{A}: H^{\infty}\left(C^{\prime}, \mathbf{C}^{n}\right) \rightarrow$ $H^{\infty}\left(A^{\prime}, \mathbf{C}^{n}\right)$ and $\mathcal{B}: H^{\infty}\left(C^{\prime}, \mathbf{C}^{n}\right) \rightarrow H^{\infty}\left(B^{\prime}, \mathbf{C}^{n}\right)$ satisfying $c=\mathcal{A} c-\mathcal{B} c$ for all $c \in$ $H^{\infty}\left(C^{\prime}, \mathbf{C}^{n}\right)$. Consider first the case when $\psi_{0}(x, u)=u$ is the identity map in the $u$ variable for each $x \in \tilde{C}$. Consider the operator

$$
\begin{aligned}
& \Phi: H^{\infty}\left(C^{\prime}, \mathbf{C}^{n}\right) \times H^{\infty}\left(\tilde{C} \times U, \mathbf{C}^{n}\right) \rightarrow H^{\infty}\left(C^{\prime}, \mathbf{C}^{n}\right), \\
& \Phi(c, \psi)(x)=\psi(x, \mathcal{A} c(x))-\mathcal{B} c(x) \quad\left(x \in C^{\prime}\right) .
\end{aligned}
$$

We claim that $\Phi$ is defined and smooth for $c \in H^{\infty}\left(C^{\prime}, \mathbf{C}^{n}\right)$ in a neighborhood of the origin and for $\psi \in H^{\infty}\left(\tilde{C} \times U, \mathbf{C}^{n}\right)$. Clearly $\Phi$ is linear and hence smooth in $\psi$. To see that $\Phi$ is smooth in $c$ we choose a neighborhood $U^{\prime} \subset \mathbf{C}^{n}$ of 0 such that $\bar{U}^{\prime} \subset U$. By Cauchy estimates the restriction map $\psi \rightarrow \psi \mid C^{\prime} \times U^{\prime}$ is a bounded linear operator from the space $H^{\infty}\left(\tilde{C} \times U, \mathbf{C}^{n}\right)$ to $\mathcal{C}^{\infty}\left(C^{\prime} \times U^{\prime}, \mathbf{C}^{n}\right)$. On the set of $c^{\prime}$ 's for which $\mathcal{A} c(x) \in U^{\prime}$ for all $x \in C^{\prime}$ (these form an open neighborhood of the origin in $H^{\infty}\left(C^{\prime}, \mathbf{C}^{n}\right)$ ) the first term in $\Phi$ is the composition operator of a linear operator $\mathcal{A}$ with a smooth map $\psi$. Hence $\Phi$ is a smooth operator. In fact we only need that $\Phi$ is of class $\mathcal{C}^{1}$ which is seen directly from the formula for its differential

$$
D \Phi(c, \psi)\left(c^{\prime}, \psi^{\prime}\right)=\psi^{\prime}(\cdot, \mathcal{A} c)+D_{2} \psi(\cdot, \mathcal{A} c) \mathcal{A} c^{\prime}-\mathcal{B} c^{\prime}
$$

Note that $\Phi\left(c, \psi_{0}\right)=\mathcal{A} c-\mathcal{B} c=c$. Hence $D_{c} \Phi\left(0, \psi_{0}\right)$ (the partial derivative of $\Phi$ with respect to the first variable) is the identity map on $H^{\infty}\left(C^{\prime}, \mathbf{C}^{n}\right)$. By the implicit function theorem in Banach spaces there is an open set $W \subset H^{\infty}\left(\tilde{C} \times U, \mathbf{C}^{n}\right)$ containing $\psi_{0}$ and a smooth map $\mathcal{C}: W \rightarrow H^{\infty}\left(C^{\prime}, \mathbf{C}^{n}\right)$ such that $\Phi(\mathcal{C}(\psi), \psi)=0$ and $\mathcal{C}\left(\psi_{0}\right)=0$. Moreover, if $\psi \in W$ satisfies $\psi(x, 0)=0$ for all $x \in \tilde{C}$, then $c=0$ solves the equation $\Phi(c, \psi)=0$ and hence by local uniqueness of solutions we have $\mathcal{C}(\psi)=0$ for any such $\psi$. The operators $\mathcal{A}^{\prime}=\mathcal{A} \circ \mathcal{C}$ and $\mathcal{B}^{\prime}=\mathcal{B} \circ \mathcal{C}$ then satisfy proposition 5.2 .

The general case (when $\psi_{0}$ is not the identity, or even close to the identity) can be reduced to the special case as follows. Since $C$ is Runge in $B$, there are open sets $\tilde{C}_{0} \subset X, B_{0} \subset X, U_{0}, U_{1} \subset \mathbf{C}^{n}$, satisfying $C \subset \tilde{C}_{0} \subset \subset \tilde{C}, 0 \in U_{0} \subset \subset U_{1} \subset \subset U, B \subset B_{0}$, such that we can approximate $\psi_{0}$ as well as desired on $\tilde{C}_{0} \times U_{1}$ by a holomorphic map $\tilde{\psi}: B_{0} \times \mathbf{C}^{n} \rightarrow \mathbf{C}^{n}$ such that $\tilde{\psi}(x, \cdot)$ is biholomorphic on $U_{1}$ for each $x \in \tilde{C}_{0}$ and $\tilde{\psi}(x, 0)=0$ for all $x \in B_{0}$. If the approximation of $\psi_{0}$ by $\tilde{\psi}$ is sufficiently close on $\tilde{C}_{0} \times U_{1}$, there is a unique holomorphic map $\psi_{0}^{\prime}: \tilde{C}_{0} \times U_{0} \rightarrow U_{1}$ which satisfies

$$
\psi_{0}(x, u)=\tilde{\psi}\left(x, \psi_{0}^{\prime}(x, u)\right) \quad\left(x \in \tilde{C}_{0}, u \in U_{0}\right)
$$

and which is so close to the map $(x, u) \rightarrow u$ on the set $\tilde{C}_{0} \times U_{0}$ that it belongs to the domain $W \subset H^{\infty}\left(\tilde{C}_{0} \times U_{0}, \mathbf{C}^{n}\right)$ of the operators $\mathcal{C}, \mathcal{A}^{\prime}$, and $\mathcal{B}^{\prime}$ obtained in the special case (with respect to the smaller set $\tilde{C}_{0} \times U_{0}$ ). We may assume that the sets $A^{\prime} \supset A, B^{\prime} \supset B$, related to the operators $\mathcal{A}^{\prime}$ resp. $\mathcal{B}^{\prime}$ as above, satisfy $B^{\prime} \subset B_{0}$ and $A^{\prime} \cap B^{\prime} \subset \subset \tilde{C}_{0}$. Note that $\mathcal{C}\left(\psi_{0}^{\prime}\right)=0$ since $\psi_{0}^{\prime}(x, 0)=0$. Moreover, for each $\psi$ which is sufficiently uniformly close to $\psi_{0}$ on $\tilde{C} \times U$ we have

$$
\psi(x, u)=\tilde{\psi}\left(x, \psi^{\prime}(x, u)\right) \quad\left(x \in \tilde{C}_{0}, u \in U_{0}\right)
$$


where $\psi^{\prime}: \tilde{C}_{0} \times U_{0} \rightarrow U_{1}$ belongs to $W$. In fact, $\psi \rightarrow \psi^{\prime}$ defines a smooth Banach space operator $\Psi: W_{0} \subset H^{\infty}\left(\tilde{C} \times U, \mathbf{C}^{n}\right) \rightarrow H^{\infty}\left(\tilde{C}_{0} \times U_{0}, \mathbf{C}^{n}\right)$ in an open neighborhood $W_{0}$ of $\psi_{0}$, with range in $W$. For each $\psi \in W_{0}$ and $\psi^{\prime}=\Psi(\psi) \in W$ the holomorphic maps $\alpha=\mathcal{A}^{\prime}\left(\psi^{\prime}\right): A^{\prime} \rightarrow \mathbf{C}^{n}$ and $\beta^{\prime}=\mathcal{B}^{\prime}\left(\psi^{\prime}\right): B^{\prime} \rightarrow \mathbf{C}^{n}$ satisfy $\psi^{\prime}(x, \alpha(x))=\beta^{\prime}(x)$ for $x \in C^{\prime}$. Hence the pair $\alpha(x)$ and $\beta(x)=\tilde{\psi}\left(x, \beta^{\prime}(x)\right.$ ) (the latter one is defined and holomorphic in $\left.B^{\prime}\right)$ satisfies

$$
\psi(x, \alpha(x))=\tilde{\psi}\left(x, \psi^{\prime}(x, \alpha(x))\right)=\tilde{\psi}\left(x, \beta^{\prime}(x)\right)=\beta(x)
$$

which is precisely (5.1). By construction $\alpha$ and $\beta$ are obtained from $\psi$ by a composition of smooth Banach space operators.

In order to reduce the proof of theorem 5.1 to proposition 5.2 we also need the following lemma. Denote by $B^{n}(\eta) \subset \mathbf{C}^{n}$ the open ball of radius $\eta$.

5.3 Lemma. Let $h: Z \rightarrow X$ be a holomorphic submersion onto a Stein manifold $X$, let $A \subset X$ be a compact set with a Stein neighborhood basis, and let $a: \tilde{A} \rightarrow Z$ be a holomorphic section defined in an open set $\tilde{A} \supset A$. Then there are an integer $n>0$, a number $\eta>0$, a Stein open set $U \subset Z$ containing $a(A)$, and a holomorphic map $\tilde{s}: U \times B^{n}(\eta) \rightarrow Z$ such that for all $z \in U$ we have

(i) $h \circ \tilde{s}(z, t)=h(z)$ for all $t=\left(t_{1}, t_{2}, \ldots, t_{n}\right) \in B^{n}(\eta)$,

(ii) $\tilde{s}(z, 0)=z$, and

(iii) the vectors $\tilde{V}_{j}(z)=\frac{\partial}{\partial t_{j}} \tilde{s}(z, 0)(1 \leq j \leq n) \operatorname{span} V T_{z}(Z)$.

Remark. Note that $\tilde{s}$ satisfies all requirements for a fiber-spray except that it is not defined globally on $U \times \mathbf{C}^{n}$.

Proof of Lemma 5.3. The set $a(A) \subset Z$ has a basis of Stein neighborhoods in $Z$ according to [Siu] and [Shd]. By Cartan's theory there exist finitely many holomorphic vector fields $\tilde{V}_{1}, \tilde{V}_{2}, \ldots, \tilde{V}_{n}$ in a neighborhood of $a(A)$ in $Z$ which are tangent to $V T(Z)$ (i.e., they are holomorphic sections of the vertical tangent bundle $V T(Z))$ and which span $V T(Z)$ at each point in the given set. Let $\theta_{j}^{t}$ be the flow of $\tilde{V}_{j}$. There is a small Stein neighborhood $U \subset Z$ of $a(A)$ and an $\eta>0$ such that the map $\tilde{s}: U \times B^{n}(\eta) \rightarrow \mathbf{C}^{n}$, given by

$$
\tilde{s}(z, t)=\tilde{s}\left(z, t_{1}, \ldots, t_{n}\right)=\theta_{1}^{t_{1}} \circ \theta_{2}^{t_{2}} \circ \cdots \circ \theta_{n}^{t_{n}}(z)
$$

satisfies all requirements.

Proof of theorem 5.1. Let $\tilde{s}: U \times B^{n}(\eta) \rightarrow Z$ be the local spray given by lemma 5.3. Choose a Stein open set $\tilde{A} \supset A$ so that $a(\tilde{A}) \subset \subset U$. Set $s_{1}(x, t)=\tilde{s}(a(x), t)$ for $x \in \tilde{A}$ and $t \in B^{n}(\eta)$. Then $s_{1}(x, 0)=a(x)$ and $s_{1}$ is a submersion along $t=0$ (i.e., a local spray onto a neighborhood of $a(\tilde{A})$ in $Z)$. Suppose that $\tilde{B} \supset B$ is an open Stein set and $b: \tilde{B} \rightarrow Z$ is a holomorphic section such that $b$ approximates $a$ in an open neighborhood $\tilde{C}$ of $C=A \cap B$. Our goal is to construct a fiber preserving holomorphic map $s_{2}: \tilde{B} \times \mathbf{C}^{n} \rightarrow Z$ such that $s_{2}(x, 0)=b(x)$ and such that $s_{2}$ is a submersion near $t=0$ which approximates $s_{1}$ in a neighborhood of $C \times\{0\}^{n}$. We then solve the equation $s_{2}(x, \psi(x, t))=s_{1}(x, t)$ to get a 
map $\psi$ as in proposition 5.2. If $\alpha$ and $\beta$ satisfy proposition 5.2 (i.e., $\psi(x, \alpha(x))=\beta(x)$ for $x$ near $C$ ) then

$$
a_{1}(x)=s_{1}(x, \alpha(x)), \quad b_{1}(x)=s_{2}(x, \beta(x)
$$

are holomorphic sections of $Z$ over neighborhoods of $A$ resp. $B$ which agree near $C=A \cap B$.

To construct $s_{2}$ we assume that $\tilde{B} \supset B$ is so small that there exists a fiber-spray $(E, p, s)$ over $Z \mid \tilde{B}=h^{-1}(\tilde{B})$. Choose a Stein open set $U^{\prime} \subset Z$ such that $a(C) \subset U^{\prime} \subset$ $\subset U \cap h^{-1}(\tilde{B})$. Let $\tilde{V}_{j}$ be the vector fields as in lemma 5.3. Since $V D s(0): E \rightarrow V T(Z)$ is a surjective vector bundle homomorphism and the set $U^{\prime}$ is Stein, we can split $E \mid U^{\prime}=$ ker $V D s(0) \oplus E^{\prime}$ and lift the sections $\tilde{V}_{j}$ to holomorphic sections $V_{j}$ of $E^{\prime} \subset E \mid U^{\prime}$ such that $V D s\left(0_{z}\right) V_{j}(z)=\tilde{V}_{j}(z)$ for all $z \in U^{\prime}$ and $1 \leq j \leq n$. For each $z \in U^{\prime}$ and each collection of $n$ vectors $\mathcal{W}=\left\{W_{1}, \ldots, W_{n}\right\} \subset E_{z}$ we define a map $s_{\mathcal{W}}(z, \cdot): \mathbf{C}^{n} \rightarrow Z_{h(z)}$ by

$$
s_{\mathcal{W}}(z, t)=s\left(\sum_{j=1}^{n} t_{j} W_{j}\right)
$$

We have $s_{\mathcal{W}}(z, 0)=z$ and $\frac{\partial}{\partial t_{j}} s_{\mathcal{W}}(z, 0)=V D s\left(0_{z}\right) W_{j}$. In particular, for the collection $\mathcal{V}(z)=\left\{V_{1}(z), \ldots, V_{n}(z)\right\}$ we get for $j=1,2, \ldots, n$ and $z \in U^{\prime}$

$$
\frac{\partial}{\partial t_{j}} s_{\mathcal{V}(z)}(z, 0)=V D s\left(0_{z}\right) V_{j}(z)=\tilde{V}_{j}(z)=\frac{\partial}{\partial t_{j}} \tilde{s}(z, 0) .
$$

The map $s_{\mathcal{W}}(z, t)$ is holomorphic in all arguments, including $\mathcal{W}$.

5.4 Lemma. Let $d$ be a metric on $Z$ and $d^{\prime}$ a metric on $E$. There are numbers $\eta>0, \delta>$ 0 with the following property. For each pair of points $z, w \in U^{\prime}$ such that $h(z)=h(w)$ and $d(z, w)<\delta$, and for each collection $\mathcal{W}=\left\{W_{1}, \ldots, W_{n}\right\} \subset E_{w}$ such that $d^{\prime}\left(W_{j}, V_{j}(w)\right)<\delta$ for $j=1, \ldots, n$, there is an injective holomorphic map $\phi_{\mathcal{W}}(z, w, \cdot): B^{n}(\eta) \rightarrow \mathbf{C}^{n}$ satisfying

(i) $s_{\mathcal{W}}\left(w, \phi_{\mathcal{W}}(z, w, t)\right)=\tilde{s}(z, t)$,

(ii) $\phi$ is holomorphic in all arguments $z, w, \mathcal{W}, t$, and

(iii) $\phi_{\mathcal{W}}(z, z, 0)=0$.

Proof. Since $U \subset Z$ is Stein, we have a splitting $U \times \mathbf{C}^{n}=M \oplus N$ where $M_{z}$ is the kernel of $D_{t} \tilde{s}(z, 0)$ (the $t$-derivative of $\tilde{s}$ at the zero section) and $N$ is some holomorphic complementary bundle. We split the fiber vectors $t=\left(t^{\prime}, t^{\prime \prime}\right) \in M_{z} \oplus N_{z}$ accordingly (so the splitting depends on the base point $z$ ). For each $z \in U$ the restriction of $\tilde{s}$ to the fiber $N_{z}$ maps a neighborhood of $0_{z} \in N_{z}$ biholomorphically onto a neighborhood of $z$ in the fiber $Z_{h(z)}$. The same is true for the restriction

$$
t^{\prime \prime} \in N_{z} \rightarrow \tilde{s}\left(z,\left(t^{\prime}, t^{\prime \prime}\right)\right) \in Z_{h(z)}
$$

of $\tilde{s}$ to fibers $\left\{t^{\prime}\right\} \oplus N_{z}$ for all sufficiently small vectors $t^{\prime} \in M_{z}$.

Now (5.2) shows that for each pair of points $z, w \in U^{\prime}$ in the same fiber $Z_{h(z)}$ which are sufficiently close together, for each sufficiently small vector $t^{\prime} \in M_{z}$, and for each collection 
of $n$ vectors $\mathcal{W}=\left\{W_{1}, \ldots, W_{n}\right\} \subset E_{w}$ which are sufficiently close to the corresponding vectors $\mathcal{V}(w)=\left\{V_{1}(w), \ldots, V_{n}(w)\right\}$, the map

$$
t^{\prime \prime} \in N_{z} \rightarrow s_{\mathcal{W}}\left(w,\left(t^{\prime}, t^{\prime \prime}\right)\right) \in Z_{h(z)}
$$

takes a neighborhood of $0_{z}^{\prime \prime} \in N_{z}$ in $N_{z}$ biholomorphically onto a neighborhood of $w$ in $Z_{h(z)}=Z_{h(w)}$ such that the image also contains the point $z$. For such choice of points and vectors we take $\phi_{\mathcal{W}}^{\prime}\left(z, w, t^{\prime}, \cdot\right): N_{z} \rightarrow N_{z}$ to be the map (5.3) followed by the (unique!) local inverse of (5.4) at $t^{\prime \prime}=0$, and then take

$$
\phi_{\mathcal{W}}\left(z, w,\left(t^{\prime}, t^{\prime \prime}\right)\right)=\left(t^{\prime}, \phi_{\mathcal{W}}^{\prime}\left(z, w, t^{\prime}, t^{\prime \prime}\right)\right)
$$

This map is defined for $t=\left(t^{\prime}, t^{\prime \prime}\right) \in M_{z} \oplus N_{z}=\mathbf{C}^{n}$ in some neighborhood of the origin $0 \in \mathbf{C}^{n}$ which we may take to be independent of $z, w, \mathcal{W}$, provided that all conditions regarding closeness are satisfied. It is easily verified that this map satisfies all required properties. Since both maps (5.3) and (5.4) depend holomorphically on all arguments, so does $\phi$.

Suppose now that $\tilde{C} \subset \tilde{A} \cap \tilde{B}$ is an open Stein set containing $C$ which is Runge in $\tilde{B}$. Assume that $b: \tilde{B} \rightarrow Z$ is a holomorphic section such that $b(\tilde{C}) \subset U^{\prime}$, where $U^{\prime} \subset Z$ is the neighborhood of $a(C)$ chosen above. We consider the restrictions $V_{j} \mid b(\tilde{C})$ as holomorphic sections of the bundle $E \mid b(\tilde{B})$ over the set $b(\tilde{C})$. By shrinking $\tilde{C}$ we get holomorphic sections $W_{j}(1 \leq j \leq n)$ of $E \mid b(\tilde{B})$ which approximate the sections $V_{j}$ uniformly on $b(\tilde{C})$ as close as desired. Write $\mathcal{W}(z)=\left\{W_{1}(z), \ldots, W_{n}(z)\right\}$ for $z=b(x) \in b(\tilde{B})$. The maps $s_{1}: \tilde{A} \times B^{n}(\eta) \rightarrow Z$ and $s_{2}: \tilde{B} \times \mathbf{C}^{n} \rightarrow Z$, given by

$$
\begin{aligned}
& s_{1}(x, t)=\tilde{s}(a(x), t), \\
& s_{2}(x, t)=s_{\mathcal{W}(b(x))}(b(x), t),
\end{aligned}
$$

are holomorphic. If $b$ is sufficiently uniformly close to $a$ over $\tilde{C}$ and if $W_{j}(b(x))$ is sufficiently close to $V_{j}(b(x))$ for each $j=1, \ldots, n$ and $x \in \tilde{C}$, then by lemma 5.4 we have for each $x \in \tilde{C}$ an injective holomorphic map

$$
\psi(x, \cdot)=\phi_{\mathcal{W}(b(x))}(a(x), b(x), \cdot): B^{n}(\eta) \rightarrow \mathbf{C}^{n}
$$

which solves the equation

$$
s_{2}(x, \psi(x, t))=s_{1}(x, t) \quad\left(x \in C^{\prime}, t \in B^{n}(\eta)\right) .
$$

Moreover, if the approximations are sufficiently close, $\psi$ is uniformly close to the map

$$
\psi_{0}(x, t)=\phi_{\mathcal{V}(a(x))}(a(x), a(x), t)
$$

which satisfies $\psi_{0}(x, 0)=0$ for $x \in \tilde{C}$. By proposition 5.3 we get open sets $A^{\prime}, B^{\prime}$, $C^{\prime}=A^{\prime} \cap B^{\prime}$ in $X$, with $A \subset A^{\prime} \subset \tilde{A}$ and $B \subset B^{\prime} \subset \tilde{B}$, and holomorphic maps $\alpha: A^{\prime} \rightarrow \mathbf{C}^{n}$, $\beta: B^{\prime} \rightarrow \mathbf{C}^{n}$, such that $\psi(x, \alpha(x))=\beta(x)$ for $x \in C^{\prime}$. The homotopies of sections

$$
a_{t}(x)=s_{1}(x, t \alpha(x)) \quad\left(x \in A^{\prime}\right), \quad b_{t}(x)=s_{2}(x, t \beta(x)) \quad\left(x \in B^{\prime}\right)
$$


for $0 \leq t \leq 1$ then satisfy theorem 5.1 .

Perhaps a word is in order regarding the proximity of the sections $a_{t}$ to $a=a_{0}$ and of $b_{t}$ to $b=b_{0}$. The rate of approximation of $\psi_{0}$ by $\psi$ depends on the proximity of $b(x)$ to $a(x)$ and on the proximity of the vector fields $\mathcal{W}(b(x))$ to the fields $\mathcal{V}(a(x))$ for $x \in \tilde{C}$. This in turn determines the estimates on the norms $\|\alpha\|_{H^{\infty}\left(A^{\prime}\right)}$ and $\|\beta\|_{H^{\infty}\left(B^{\prime}\right)}$ (by proposition 5.2). Since the map $s_{1}$ (5.5) only depends on the section $a$, the definition (5.6) shows that the estimate of $d\left(a_{t}(x), a(x)\right)$ for $x \in A^{\prime}$ and $t \in[0,1]$ depends only on $\|\alpha\|_{H^{\infty}\left(A^{\prime}\right)}$, and we get the stated approximation result over $A^{\prime}$. However, the map $s_{2}$ (5.5) depends both on $b$ and on $\mathcal{W}$. These quantities are under control only on $\tilde{C}$ and not on all of $\tilde{B}$ (since we apply Runge approximation). Therefore we can estimate $d\left(b_{t}(x), b(x)\right)$ in terms of $\|\beta\|_{H^{\infty}\left(B^{\prime}\right)}$ only for points $x \in C^{\prime}$ and not on $B^{\prime} \backslash C^{\prime}$.

The following is an extension of theorem 5.1 to parametrized families of sections.

5.5 Theorem. Let $h: Z \rightarrow X$ be a holomorphic submersion onto a Stein manifold $X$, let $d$ a metric on $Z$, and let $(A, B)$ be a Cartan pair in $X$ (def. 2.3) such that the set $C=A \cap B$ is Runge in $B$. Suppose that $\tilde{B}$ is an open neighborhood of $B$ in $X$ such that the restriction $Z \mid \tilde{B}=h^{-1}(\tilde{B})$ admits a fiber-spray (def. 3.1). Let $Y$ be a compact Hausdorff space (the parameter space) and $Y_{0} \subset Y$ a compact subset. Let $\tilde{A} \subset X$ be an open neighborhood of $A$ and $a: \tilde{A} \times Y \rightarrow Z$ a continuous map such that for each $y \in Y$, $a(\cdot, y)$ is a holomorphic section of $Z$ over $\tilde{A}$. Then for each $\epsilon>0$ there is a $\delta>0$ satisfying the following property. If $b: \tilde{B} \times Y \rightarrow Z$ is a continuous map such that for each $y \in Y$, $b(\cdot, y)$ is a holomorphic section of $Z$ over $\tilde{B}$ satisfying

$$
\begin{array}{ll}
d(a(x, y), b(x, y))<\delta & (x \in \tilde{C}=\tilde{A} \cap \tilde{B}, \quad y \in Y), \\
a(x, y)=b(x, y) & \left(x \in \tilde{C}, \quad y \in Y_{0}\right),
\end{array}
$$

then there exist smaller neighborhoods $A^{\prime} \supset A, B^{\prime} \supset B$ and homotopies $a_{t}: A^{\prime} \times Y \rightarrow Z$ resp. $b_{t}: B^{\prime} \times Y \rightarrow Z(0 \leq t \leq 1)$ of families of holomorphic sections such that $a_{0}=a$, $b_{0}=b, a_{1}=b_{1}$ on $C^{\prime}=A^{\prime} \cap B^{\prime}$, and for each $0 \leq t \leq 1$ we have

$$
\begin{array}{ll}
d\left(a_{t}(x, y), a(x, y)\right)<\epsilon & \left(x \in A^{\prime}, y \in Y\right) \\
d\left(b_{t}(x, y), b(x, y)\right)<\epsilon & \left(x \in C^{\prime}, y \in Y\right) \\
a_{t}(x, y)=b_{t}(x, y) & \left(x \in C^{\prime}, y \in Y_{0}\right)
\end{array}
$$

Proof. This can done by essentially repeating the proof of theorem 5.1 with the addition of the parameter $y$. We shall only indicate a few critical places in the proof where it is not completely obvious what must be done. First one needs for each $y \in Y$ holomorphic maps

$$
s_{1, y}: \tilde{A} \times B^{n}(\eta) \rightarrow Z, \quad s_{2, y}: \tilde{B} \times \mathbf{C}^{n} \rightarrow Z
$$

for some integer $n>0$ and some $\eta>0$ which are related to the sections $a_{y}=a(\cdot, y)$ resp. $b_{y}=b(\cdot, y)$ as in $(5.5)$ and which depend continuously on the parameter $y \in Y$. To get 
$s_{1, y}$ we cover $Y$ by finite number of open sets $Y_{j}(1 \leq j \leq J)$ such that for each $j$ there is an open Stein set $U_{j} \subset Z$, with $a_{y}(\tilde{A}) \subset D_{j}$ for each $y \in Y_{j}$, and there are finitely many holomorphic vector fields $\tilde{V}_{k}^{j}\left(1 \leq k \leq k_{j}\right)$ which generate $V T(Z)$ at each point of $U_{j}$. (This is similar to the proof of lemma 4.4 above.) Let $\chi_{j}: Y \rightarrow[0,1](1 \leq j \leq J)$ be a continuous partition of the unity on $Y$ subordinate to the covering $\left\{Y_{j}\right\}$. Then the vector fields $\chi_{j}(y) \tilde{V}_{k}^{j}\left(1 \leq j \leq J, 1 \leq k \leq k_{j}\right)$ are well defined and holomorphic in a neighborhood of the section $a_{y}(\tilde{A})$ for each $y \in Y$ (since $\chi_{j}(y)=0$ for those $y \in Y$ for which the field $\tilde{V}_{k}^{j}$ is not defined near $a_{y}(\tilde{A})$, i.e., for $y$ outside $\left.Y_{j}\right)$. Together these $n$ fields generate the vertical tangent bundle $V T(Z)$ at each point $a_{y}(x)$ for $x \in \tilde{A}$ and $y \in Y$. Using these fields and their local flows we get as before a family of submersions $s_{1, y}(5.7)$ depending continuously on $y \in Y$.

With a similar argument (see lemma 4.4) we obtain a family of maps $s_{2, y}(5.7)$ which are submersions in a neighborhood of the zero section over the set $\tilde{C}$ and which approximate $s_{1, y}$ near $\tilde{C} \times\{0\}^{n}$. This gives a family of transition mappings $\psi_{y}$ as in proposition 5.2 which are continuous in $y$ and approximate a certain initial family $\psi_{0, y}$. By proposition 5.2 we obtain families of bounded holomorphic maps $\alpha_{y}: A^{\prime} \rightarrow \mathbf{C}^{n}, \beta_{y}: B^{\prime} \rightarrow \mathbf{C}^{n}$, depending continuously on $y \in Y$ and satisfying

$$
\psi_{y}\left(x, \alpha_{y}(x)\right)=\beta_{y}(x) \quad\left(x \in C^{\prime}, y \in Y\right) .
$$

This gives for all $y \in Y$ homotopies of sections

$$
a_{t}(x, y)=s_{1, y}\left(x, t \alpha_{y}(x)\right) \quad\left(x \in A^{\prime}\right), \quad b_{t}(x, y)=s_{2, y}\left(x, t \beta_{y}(x)\right) \quad\left(x \in B^{\prime}\right) .
$$

Moreover, for $y \in Y_{0}$ (when the sections $a_{y}$ and $b_{y}$ agree over $\tilde{C}$ and hence define a section over a neighborhood of $A \cup B)$ we have by construction $\psi_{y}(x, 0)=0$ for all $x \in \tilde{C}$. Therefore $\alpha_{y}=0$ and $\beta_{y}=0$ for such $y$ (prop. 5.2), and hence the above homotopies are fixed for $y \in Y_{0}$ as required. Everything else is clear from the earlier arguments.

5.6 Corollary. Let $X$ be a Stein manifold and $A \subset A^{\prime}$ a non-critical strongly pseudoconvex extension in $X$ (def. 2.1). Let $F$ be a complex manifold with a spray and $Z=X \times F \rightarrow X$ the associated trivial bundle with fiber $F$. Let $d$ be a metric on $Z$. Given a homotopy $a_{t}(0 \leq t \leq 1)$ of holomorphic sections of $Z$ in a neighborhood of $A$ such that $a_{0}$ and $a_{1}$ are holomorphic in a neighborhood of $A^{\prime}$, there is for each $\epsilon>0$ a homotopy $\tilde{a}_{t}$ $(0 \leq t \leq 1)$ of holomorphic sections in a neighborhood of $A^{\prime}$ such that $\tilde{a}_{0}=a_{0}, \tilde{a}_{1}=a_{1}$, and

$$
d\left(\tilde{a}_{t}(x), a_{t}(x)\right)<\epsilon \quad(x \in A, 0 \leq t \leq 1) .
$$

Proof. By theorem 2.5 there is a finite sequence

$$
A=A_{0} \subset A_{1} \subset A_{2} \subset \ldots \subset A_{k}=A^{\prime}
$$

such that for each $j=0,1, \ldots, k-1$ we have $A_{j+1}=A_{j} \cup B_{j}$, where $\left(A_{j}, B_{j}\right)$ is a convex bump. Suppose inductively that we have already approximated the initial homotopy $a_{t}=$ 
$a_{t}^{0}$ on $A$ by a homotopy $a_{t}^{j}(0 \leq t \leq 1)$ consisting of sections that are holomorphic in a neighborhood of $A_{j}$ (with $a_{0}^{j}=a_{0}$ and $a_{1}^{j}=a_{1}$ ). Since $B_{j}$ is a convex bump on $A_{j}$, we can apply theorem 4.5 to approximate the homotopy $a_{t}^{j}$ in a neighborhood of $C_{j}=A_{j} \cap B_{j}$ by a homotopy $b_{t}$ consisting of holomorphic sections in a neighborhood of $B_{j}$ and such that $b_{0}=a_{0}, b_{1}=a_{1}$. If the approximation is sufficiently close, we can apply theorem 5.5 to glue the families $a_{t}^{j}$ and $b_{t}$ over $C_{j}$ into a single family $a_{t}^{j+1}(0 \leq t \leq 1)$ consisting of sections which are holomorphic in a neighborhood of $A_{j+1}=A_{j} \cup B_{j}$, which equal $a_{0}$ resp. $a_{1}$ when $t=0$ resp. $t=1$, and which approximate the sections $a_{t}^{j}$ on $A_{j}$. This completes the induction step. In a finite number of steps we reach a desired homotopy $\tilde{a}_{t}=a_{t}^{k}$ satisfying corollary 5.6.

Remark. The proof of corollary 5.6 remains valid if the bundle $Z$ is trivial over a neighborhood of each bump $B_{j}$ but not necessarily over $A^{\prime}$. Thus the corollary provides an extension of the h-principle from $A$ to any non-critical strongly pseudoconvex extension of $A$.

\section{\&6. Extending holomorphic sections across pseudoconvex bumps.}

In this section we prove theorem 2.6, thereby concluding the proof of theorems 1.3 and 1.4. We present the proof in the case without parameters, but all arguments go through in the general parametric case by using suitable versions of the approximation and gluing theorems which were proved in sections 4 and 5. There are three main steps:

- approximate the given section $a$ in a neighborhood of $C=A \cap B$ by a section $b$ which is holomorphic in a neighborhood of $B$;

- glue the sections $a$ and $b$ by theorem 5.1 (or theorem 5.5) to obtain a holomorphic section $\tilde{a}$ in a neighborhood of $A \cup B$;

- show that the new section $\tilde{a}$ is homotopic to $a$ and satisfies all required properties.

We present all arguments in the general case when $(A, B)$ is a pseudoconvex bump and the set $C=A \cap B$ is not necessarily contractible. In the special case of convex bumps the arguments involving homotopies can be substantially simplied by using contractibility of $C$ and the fact that $A$ is a strong deformation retraction of $A \cup B$.

6.1 Proposition. There exists a homotopy $b_{t}(0 \leq t \leq 1)$ of continuous sections in a neighborhood of $B$ such that $b_{0}$ is holomorphic in a neighborhood of $B$, each $b_{t}$ is holomorphic in a neighborhood of $C$, and $b_{1}=a$.

Proof. Since $B$ is star-shaped, it has an open contractible neighborhood $\tilde{B}$. By hypothesis we may choose $\tilde{B}$ so small that $Z \mid \tilde{B}$ is a trivial bundle. Hence there is a holomorphic section $b_{0}$ of $Z$ over $\tilde{B}$ (we may simply take a constant section in a given trivialization) and a homotopy $b_{t}^{\prime}: \tilde{B} \rightarrow Z(0 \leq t \leq 1)$ of continuous sections connecting $b_{0}^{\prime}=b_{0}$ and $b_{1}^{\prime}=a$ (such a homotopy exists since $\tilde{B}$ is contractible and the bundle is trivial over $\tilde{B}$ ).

Recall (part (v) in def. 2.2) that we have a strongly plurisubharmonic function $\tau \geq 0$ in a neighborhood of $C=A \cap B$ such that $S=\{\tau=0\}$ is a totally real sphere contained in 
an affine plane $T$ (in some holomorphic coordinates in a neighborhood of $B$ ), $C=\{\tau \leq 1\}$, and $\tau$ has no critical points on $C \backslash S$. Our first goal is to modify the homotopy $b_{t}^{\prime}$ so as to make it holomorphic in a neighborhood of $S$. Assume (as we may) that $S$ is realanalytic. Let $T^{\prime}$ be the affine totally real subspace of maximal dimension containing $T$ (and hence $S$ ). We can approximate $b_{t}^{\prime} \mid S$ uniformly on $S$ by a homotopy consisting of real-analytic sections over a neighborhood of $B$ without changing the sections $b_{0}^{\prime}=b_{0}$ and $b_{1}^{\prime}=a$. We still denote this real-analytic homotopy by $b_{t}^{\prime}$. For each $t$ the section $b_{t}^{\prime} \mid T^{\prime}$ extends (by complexification) to a unique holomorphic section $\tilde{b}_{t}$ in a neighborhood of $T^{\prime} \cap C$ (independent of $t$ ). Hence $\tilde{b}_{t}$ is a holomorphic homotopy in a neighborhood of $S_{0}=\left\{\tau \leq c_{0}\right\}$ for some sufficiently small $c_{0}>0$. Of course this process does not affect the sections that were already holomorphic, so we have $\tilde{b}_{0}=b_{0}$ and $\tilde{b}_{1}=a$ on $S_{0}$.

Since $C$ is a non-critical strongly pseudoconvex extension of $S_{0}$, corollary 5.6 implies that $\tilde{b}_{t}$ can be approximated uniformly on $S_{0}$ by another homotopy $b_{t}(0 \leq t \leq 1)$ which is holomorphic in a neighborhood of $C$ and connects $b_{0}$ and $a$.

To complete the proof we must show that the homotopy $b_{t}$ (which has so far been defined and holomorphic in a neighborhood of $C$ ) extends to a continuous homotopy from $b_{0}$ to $a$ in a neighborhood of $B$. To do this we first reparametrize both homotopies $b_{t}$ and $b_{t}^{\prime}$ so that for some small $\delta>0$ we have

$$
b_{t}=b_{t}^{\prime}=b_{0} \quad \text { for } 0 \leq t \leq \delta, \quad b_{t}=b_{t}^{\prime}=a \quad \text { for } 1-\delta \leq t \leq 1 .
$$

6.2 Lemma. If $b_{t} \mid S$ is sufficiently uniformly close to $b_{t}^{\prime} \mid S$ on $S=\{\tau=0\} \subset C$ for each $t \in[0,1]$, and if (6.1) holds for some $\delta>0$, then there exist a neighborhood $\tilde{C}$ of $C$ and a two-parameter homotopy $\tilde{b}_{t, s}(0 \leq t, s \leq 1)$ of continuous sections of $Z$ over $\tilde{C}$ satisfying

$$
\begin{aligned}
& \tilde{b}_{t, 0}=b_{t}, \quad \tilde{b}_{t, 1}=b_{t}^{\prime} \text { for } 0 \leq t \leq 1 \\
& \tilde{b}_{t, s}=b_{t}=b_{t}^{\prime} \text { for } t \in\{0,1\} \text { and } 0 \leq s \leq 1 .
\end{aligned}
$$

Proof. If $b_{t} \mid S$ is sufficiently uniformly close to $b_{t}^{\prime} \mid S$ for each $t \in[0,1]$ (which we may assume to be the case), we can use the spray as in lemma 3.2 to obtain a two parameter homotopy of sections $b_{t, s}: S \rightarrow Z(0 \leq t, s \leq 1)$ satisfying

$$
\begin{aligned}
& b_{t, 0}=b_{t}\left|S, \quad b_{t, 1}=b_{t}^{\prime}\right| S \text { for } 0 \leq t \leq 1 \\
& b_{t, s}=b_{t}=b_{t}^{\prime} \text { for } t \in[0, \delta] \cup[1-\delta, 1] \text { and } 0 \leq s \leq 1 .
\end{aligned}
$$

Furthermore, $S$ is a strong deformation retraction of a neighborhood $\tilde{C}$ of $C$, i.e., there is a smooth family of maps $\Theta_{t}: \tilde{C} \rightarrow \tilde{C}(0 \leq t \leq 1)$ such that $\Theta_{0}$ is the identity on $\tilde{C}$, each $\Theta_{t}$ is the identity on $S$, and $\Theta_{1}(\tilde{C})=S$. Choose a smooth function $u:[0,1] \rightarrow[0,1]$ which is zero in a neighborhood of $t=0$ and $t=1$ and is identically one on $[\delta, 1-\delta]$. We identify sections of $Z$ over $\tilde{C}$ (or over $\tilde{B}$ ) by mappings into the fiber $F$, using the triviality of $Z \mid \tilde{B}$. For each $x \in \tilde{C}$ and $0 \leq t \leq 1$ we define

$$
\tilde{b}_{t, s}(x)= \begin{cases}b_{t}\left(\Theta_{3 s u(t)}(x)\right) ; & \text { if } 0 \leq s \leq 1 / 3 \\ b_{t, 3 s-1}\left(\Theta_{u(t)}(x)\right) ; & \text { if } 1 / 3 \leq s \leq 2 / 3 \\ b_{t}^{\prime}\left(\Theta_{3(1-s) u(t)}(x)\right) ; & \text { if } 2 / 3 \leq s \leq 1\end{cases}
$$


Clearly these maps are defined for all $x \in \tilde{C}$. We have $\tilde{b}_{t, 0}=b_{t} \circ \Theta_{0}=b_{t}$ and $\tilde{b}_{t, 1}=$ $b_{t}^{\prime} \circ \Theta_{0}=b_{t}^{\prime}$. Moreover, when $t$ is near 0 or near 1 , or when $x \in S$, we have $\tilde{b}_{t, s}(x)=b_{t, s}(x)$ for all $s \in[0,1]$. Thus $\tilde{b}_{t, s}$ satisfies lemma 6.2.

Choose a smooth function $v: X \rightarrow[0,1]$ such that $v=1$ in a neighborhood $C^{\prime} \subset \tilde{C}$ of $C$ and $\operatorname{supp} v \subset \subset \tilde{C}$. Let $\tilde{b}_{t, s}$ be as in lemma 6.2. Consider the homotopy

$$
\tilde{b}_{t, 1-v(x)}(x) \quad(x \in \tilde{B}, 0 \leq t \leq 1) .
$$

By the choice of $v$ this equals $\tilde{b}_{t, 0}(x)=b_{t}(x)$ for $x \in C^{\prime}$ (so it is holomorphic there), it is defined for all $x \in \tilde{B}$, and it equals $b_{t}^{\prime}(x)$ for $x$ outside $\tilde{C}$. This homotopy, which we again denote by $b_{t}$, satisfies proposition 6.1 .

In what follows we shall shrink the neighborhoods $\tilde{B}$ and $\tilde{C}$ several times without mentioning this again, and without changing the notation. We are now in position to apply the h-Runge approximation (theorem 4.1 or 4.2 ) to approximate the homotopy $b_{t}$ from proposition 6.1 uniformly in $\tilde{C}$ by a holomorphic homotopy $\tilde{b}_{t}: \tilde{B} \rightarrow Z$ such that $\tilde{b}_{0}=b_{0}$. The section $b=\tilde{b}_{1}$ then approximates $a$ in $\tilde{C}$ as well as desired. Hence by theorem 5.1 (or 5.5) we can glue $a$ and $b$ into a single section $\tilde{a}$ which is holomorphic in a neighborhood $A^{\prime}$ of $A \cup B$ and which approximates $a$ in a neighborhood of $A$.

It remains to show that there is a homotopy of sections $a_{t}(0 \leq t \leq 1)$ in a neighborhood of $A \cup B$, connecting $a_{0}=a$ and $a_{1}=\tilde{a}$, such that each $a_{t}$ is holomorphic in a neighborhood of $A$ and approximates $a$ there (so $a_{t}$ will satisfy theorem 2.6). In a neighborhood of $A$ such a homotopy is provided by theorem 5.1. Our goal is to extend this homotopy to a neighborhood of $A \cup B$ by modifying it outside some neighborhood of $A$.

Recall that over $\tilde{B}$ we have the following homotopies:

(i) the homotopy $b_{t}$ from $b_{0}$ to $b_{1}=a$, given by proposition 6.1 ;

(ii) the homotopy $\tilde{b}_{t}$ from $\tilde{b}_{0}=b_{0}$ to $\tilde{b}_{1}=b$, obtained by approximating $b_{t}$ in $\tilde{C}$;

(iii) the homotopy $b_{t}^{\prime}$ from $b_{0}^{\prime}=b\left(=\tilde{b}_{1}\right)$ to $b_{1}^{\prime}=\tilde{a}$, given by theorem 5.1.

Note that all these homotopies are holomorphic in $\tilde{C}$ and $b_{t}^{\prime}$ approximates $b$ (and hence $a$ ) there. If we combine these three homotopies in the correct order (first follow $b_{1-t}$ from $a$ to $b_{0}$, then follow $\tilde{b}_{t}$ from $b_{0}$ to $\tilde{b}_{1}=b$, and finally follow $b_{t}^{\prime}$ from $b$ to $\tilde{a}$ ), we get a homotopy from $a$ to $\tilde{a}$ over $\tilde{B}$ which is holomorphic over $\tilde{C}$. However, we must show that this homotopy over $\tilde{B}$ can be glued with the homotopy $a_{t}$ into a single homotopy from $a$ to $\tilde{a}$ in a neighborhood of $A \cup B$.

In order to do this we will first join the above homotopies (i)-(iii) over $\tilde{B}$ into a new homotopy $h_{t}$ from $a$ to $\tilde{a}$ which in addition will approximates $a$ in $\tilde{C}$. For convenience we shall define $h_{t}$ initially on the $t$-interval $[0,4]$ and subsequently rescale the parameter to $[0,1]$. According to the remark following theorem 4.1 we may assume that there is a two-parameter homotopy $g_{t, s}: \tilde{B} \rightarrow Z(0 \leq t, s \leq 1)$ which is holomorphic in $\tilde{C}$ and satisfies

$$
g_{t, 0}=b_{t}, \quad g_{t, 1}=\tilde{b}_{t}, \quad g_{0, s}=b_{0},
$$


and such that $g_{1, s}$ approximates $b_{1}=a$ in $\tilde{C}$ for each $s \in[0,1]$. Choose a smooth function $\chi$ on $X$ with values in $[0,1]$ such that $\chi=1$ in a neighborhood of $A$ and $\chi=0$ in a neighborhood of $B \backslash \tilde{C}$. Such $\chi$ exists since the sets $A \backslash B$ and $B \backslash A$ are separated. We now define for each $x \in \tilde{B}$

$$
h_{t}(x)= \begin{cases}b_{1-t+t \chi(x)}(x), & \text { if } 0 \leq t \leq 1 \\ g_{\chi(x), t-1}(x), & \text { if } 1 \leq t \leq 2 \\ \tilde{b}_{\chi(x)+(1-\chi(x))(t-2)}(x), & \text { if } 2 \leq t \leq 3 \\ b_{t-3}^{\prime}(x), & \text { if } 3 \leq t \leq 4\end{cases}
$$

The reader may verify that this is indeed a homotopy from $h_{0}=a$ to $h_{4}=\tilde{a}$. For $x$ in a neighborhood of $C$ we have $\chi(x)=1$ and hence

$$
h_{t}(x)= \begin{cases}b_{1}(x)=a(x), & \text { if } 0 \leq t \leq 1 \\ g_{1, t-1}(x), & \text { if } 1 \leq t \leq 2 \\ \tilde{b}_{1}(x)=b(x), & \text { if } 2 \leq t \leq 3 \\ b_{t-3}^{\prime}(x), & \text { if } 3 \leq t \leq 4\end{cases}
$$

Hence $h_{t}$ is holomorphic near $C$ and approximates $a$ there (since all homotopies in question are close to $a$ on $\tilde{C}$ ). Hence we may assume that on $\tilde{C}$ both homotopies $a_{t}$ and $h_{t}$ (which we rescale to the $t$-interval $[0,1]$ ) approximate $a$ so well that their images (over $\tilde{C}$ ) belong to a tubular neighborhood of $a(\tilde{C}) \subset Z$ in which we can apply lemma 3.2. This means that we can view these sections on $\tilde{C}$ as sections of a certain holomorphic vector bundle over $\tilde{C}$. This allows us to find a two parameter homotopy $k_{t, s}$ joining $a_{t}$ and $h_{t}$ over $\tilde{C}$ (we can simply use the convex combinations of the two sections in the given vector bundle.) Finally we patch $a_{t}$ and $h_{t}$ using $k_{t, s}$ into the homotopy

$$
\tilde{a}_{t}(x)=k_{t, 1-\chi(x)}(x)
$$

where $\chi$ is a smooth function chosen as above. For $x$ near $A$ we have $\chi(x)=1$ and hence $\tilde{a}_{t}(x)=k_{t, 0}(x)=a_{t}(x)$, while for $x$ near $B \backslash \tilde{A}$ we have $\chi(x)=0$ ad hence $\tilde{a}_{t}(x)=k_{t, 1}(x)=$ $h_{t}(x)$. We denote this new homotopy again $a_{t}$.

Finally we choose a smooth function $\eta$ on $X$ with values in $[0,1]$ such that $\eta=1$ near $A \cup B$ and $\operatorname{supp} \eta \subset A^{\prime}$, where $A^{\prime}$ is the neighborhood of $A \cup B$ on which the homotopy $a_{t}$ has been defined. The homotopy $a_{t \eta(x)}(x)(0 \leq t \leq 1)$ is now defined for all $x \in X$, it equals $a_{0}(x)=a(x)$ for $x \in X \backslash A^{\prime}$ and $t \in[0,1]$, and it equals $a_{t}(x)$ for $x \in A \cup B$. This completes the proof of theorem 2.6.

\section{References.}

[AFR] P. Ahern, M. Flores, J.-P. Rosay: On $\mathbf{R}^{+}$and $\mathbf{C}$ complete holomorphic vector fields. Proc. Amer. Math. Soc., to appear.

[Car] H. Cartan: Espaces fibrés analytiques. Symposium Internat. de topologia algebraica, Mexico, 97-121 (1958). (Also in Oeuvres, vol. 2, Springer, New York, 1979.) 
[BFo] G. Buzzard, J. E. Fornæss: An embedding of $\mathbf{C}$ in $\mathbf{C}^{2}$ with hyperbolic complement. Math. Ann. 306, 539-546 (1996).

[Chi] E. M. Chirka: Complex analytic sets. Mathematics and its Applications (Soviet Series), 46, Kluwer, Dordrecht, 1989.

[Dem] J.-P. Demailly: Un exemple de fibré holomorphe non de Stein à fibre $\mathbf{C}^{\mathbf{2}}$ ayant pour base le disque ou le plan. Invent. Math. 48, 293-302 (1978).

[Eli] Y. Eliashberg: Topological characterization of Stein manifolds of dimension $>2$. Internat. J. Math. 1, 29-46 (1990).

[EGr] Y. Eliashberg, M. Gromov: Embeddings of Stein manifolds. Ann. Math. 136, 123-135 (1992).

[For] O. Forster: Topologische Methoden in der Theorie der Steinscher Räume. (Iternat. Congress in Math., Nice, 1970, pp. 613-618) Gauthier-Villars, Paris, 1971.

[FR1] O. Forster and K. J. Ramspott: Okasche Paare von Garben nicht-abelscher Gruppen. Invent. Math. 1, 260-286 (1966).

[FR2] O. Forster and K. J. Ramspott: Analytische Modulgarben und Endromisbündel. Invent. Math. 2, 145-170 (1966).

[Fo1] F. Forstnerič: Actions of $(\mathbf{R},+)$ and $(\mathbf{C},+)$ on complex manifolds. Math. Z. 223, 123-153 (1996).

[Fo2] F. Forstnerič: Interpolation by holomorphic automorphisms and embeddings in $\mathbf{C}^{n}$. J. Geom. Anal. 9, no.1, (1999) 93-118.

[FGR] F. Forstnerič, J. Globevnik, J.-P. Rosay: Non straightenable complex lines in $\mathbf{C}^{2}$. Arkiv Mat. 34, 97-101 (1996).

[FP] F. Forstnerič and J. Prezelj: Oka's principle for holomorphic submersions with sprays. Preprint, 1999.

[Gr1] H. Grauert: Charakterisierung der holomorph vollständigen Räume. Math. Ann. 129, 233-259 (1955).

[Gr2] H. Grauert: Approximationssätze für holomorphe Funktionen mit Werten in komplexen Räumen. Math. Ann. 133, 139-159 (1957).

[Gr3] H. Grauert: Holomorphe Funktionen mit Werten in komplexen Lieschen Gruppen. Math. Ann. 133, 450-472 (1957).

[Gr4] H. Grauert: Analytische Faserungen ber holomorph-vollstndigen Rumen. Math. Ann. 135, 263-273 (1958).

[GRe] H. Grauert, R. Remmert: Theory of Stein Spaces. Grundl. Math. Wiss. 227, Springer, New York, 1977.

[Gro] M. Gromov: Oka's principle for holomorphic sections of elliptic bundles. J. Amer. Math. Soc. 2, 851-897 (1989).

[GRo] C. Gunning, H. Rossi: Analytic functions of several complex variables. PrenticeHall, Englewood Cliffs, 1965.

$[\mathrm{HKu}]$ P. Heinzner and F. Kutzschebauch: An equivariant version of Grauert's Oka principle. Invent. Math. 119, 317-346 (1995). 
[HL1] G. M. Henkin, J. Leiterer: Theory of functions on complex manifolds. AkademieVerlag, Berlin, 1984.

[HL2] G. Henkin, J. Leiterer: Proof of Oka-Grauert principle without the induction over basis dimension. Preprint, Karl Weierstrass Institut für Mathematik, Berlin, 1986.

[HL3] G. Henkin, J. Leiterer: The Oka-Grauert principle without induction over the basis dimension. Math. Ann. 311, 71-93 (1998).

[Hö1] L. Hörmander: $L^{2}$ estimates and existence theorems for the $\bar{\partial}$ operator. Acta Math. 113, 89-152 (1965).

[Hö2] L. Hörmander: An Introduction to Complex Analysis in Several Variables, 3rd ed. North Holland, Amsterdam, 1990.

[Lei] J. Leiterer: Holomorphic Vector Bundles and the Oka-Grauert Priciple. Encyclopedia of Mathematical Sciences, vol. 10, 63-103; Several Complex Variables IV, Springer, 1989.

[Ram] K. J. Ramspott: Stetige und holomorphe Schnitte in Bundeln mit homogener Faser. Math. Z. 89, 234-246 (1965).

[Ros] J.-P. Rosay: A counterexample related to Hartogs phenomenon (a question by E. Chirka). Michigan Math. J. 45, 529-535 (1998).

[RRu] J.-P. Rosay, W. Rudin: Holomorphic maps from $\mathbf{C}^{n}$ to $\mathbf{C}^{n}$. Trans. Amer. Math. Soc. 310, 47-86 (1988)

[Shd] M. Schneider: Tubenumgebungen Steinscher Räume. Manuscripta Math. 18, 391-397 (1976).

[Sch] J. Schürmann: Embeddings of Stein spaces into affine spaces of minimal dimension. Math. Ann. 307, 381-399 (1997).

[Siu] Y.T. Siu: Every Stein subvariety admits a Stein neighborhood. Invent. Math. 38, 89-100 (1976).

[Ste] K. Stein: Analytische Funktionen mehrerer komplexer Vernderlichen zu vorgegebenen Periodizittsmoduln und das zweite Cousinsche Problem. Math. Ann. 123, 201-222 (1951).

Franc Forstnerič

Department of Mathematics

University of Wisconsin

Madison, WI 53706, USA

Current address:

IMFM, University of Ljubljana

Jadranska 19

1000-Ljubljana, Slovenia
Jasna Prezelj

Faculty of Mechanical Engineering University of Ljubljana

Aškerčeva 6

1000-Ljubljana, Slovenia 